\title{
Die Spezifität der Tuberkulinreaktion.
}

\section{Vergleichende Untersuchungen mit Tuberkulin und Eiweiß- körpern an experimentellem und klinischem Material.}

\author{
Von \\ Dr. Ericl Hagemann. \\ (Aus der Medizinischen Klinik Kiel [Direktor: Prof. Dr. A. Schittenhelm].) \\ Eingegangen am 2. Juni 1929.
}

Eine Arbeit über die Spezifität des Tuberkulins wird zweckmäBig mit einer kurzen Definition des Begriffs beginnen müssen, so überflüssig dies bei einer so allgemein bokannten und gebrauchten Vorstellung anmuten mag. Ein Überblick aber über die seit 3 Jahrzehnten entstandene Literatur dieser Frage rechtfertigt dieses Vorgehen; scheint doch gleichsinnig mit dor Häufigkeit der Anwendung eines solchen Begriffs seine Anschaulichkeit zu verblassen.

Unter spezifischer Wirkung des Tuberkulins vorstehen wir zunächst, daß sie sich allein auf don tuberkulösen Organismus beschränkt und den gesunden uubeeinflußt lïßt. Letzteres wird heute fast allgemein zugegoben (bewiesen durch Untersuchungen an sicher tuberkulosefreien Individuen. Engel und Bauer, Ruppel u. a.).

Ferner mul gefordert werdon, daß das Tuberkulin in einzigartiger Weise imstande ist, im tuberkulösen Herd wie im Gesamtorganismus des Kranken charakteristische Veränderungen hervorzurufen, dio auf Grund einer spezifischen Ċberempfindlichkeit (Allergie) auftreten.

Wäre irgendein andrer Stoff, ein Eiweißkörper z. B., imstande, ,Tuberkulinreaktionon" in gleicher Weise auszulösen, dürften wir. den Ausdruck der Spezifität auf das Tuborkulin allerdings nicht mehr anwenden.

Seit den ersten Beobachtumgen über die Wirkungen des Tuberkulins auf den tuberkulösen Organismus, insbesondere den tuberkulösen Herd, sind auch Zweifel äher ihre Spezifität laut geworden. Auf dem $X$. KongreB für iunere Medizin (1891) botonte Ziegler, daß die Prozesse, die sich in der Tmgebung des Tuberkels infolge der $K o c h s c h e n$ Injektionen abspielen, keine Vorgänge sind. die etwas ganz Besonderes darbieten, und im Vorlaufe der durch Tuberkulin nicht beeinflußten Tubcrkulose fehlen. Die Fntzündungsprozesse, die auch sonst in der Umgebung des Tuberkels vorhanden sind, werden durch dic Injektion gesteigert. bzw. sie 1reten in ganz akuter und vielleicht intensiverer Weise auf als unter gewölnnlichen Verhälnissen. Wie bei unbeeinfluBtem Ver- 
lauf kann dieser Vorgang in Richtung der Heilung oder Verschlimmerung ausschlagen, aber rascher und energischer.

Damit wird also die Tuberkulinreaktion als Reizerscheinung in den Rahmen natürlicher Krankheitsvorgänge eingeordnet -.. eine Vorstellung, die uns heute ganz zu eigen ist; mögen auch die realitiven Prozesse, die sich dabei abspielen, in einzelnen der Gegenstand der mannigfachsten Kontroverse gewesen sein. Heute nähern wir uns mit der Auffassung des Tuberkulins als Reizstoff, wie sie am prägnantesten wohl durch Selter vertreten wird, fast vollkommen jener vor 30 Jahren ausgesprochenen Vorstellung.

Diese Fähigkeit des Tuberkulins, Reaktionen zu erzeugen, beruht nach fast allgemein gültiger Anschauung auf der spezifischen Uberempfindlichkeit, der Allergie v. Pirquet's, des tuberkulösen Herdes wie aller seheinbar un. beteiligten Körperzellen, einer biologischen Umstimmung mit fein abgestimmter Reaktion auf adäquate Reize. Andere Stoffe können außerhalb des Herdes im tuberkulösen Organismus diese charakteristischen Reaktionen, z. B. in der Haut, nicht erzeugen (Bessau. Sons und v. Mikulicz-Radecki). Es ist bewiesen, daß Tuberkulininjektionen histologisch ein andres Bild machen als Cutanreaktionen anderer Stoffe (Klingmüller, Zieler, Doutrelepont), durch Neuinjektionen (auch von Eiweißkörpern) oder Verändorungen im Krankheitsverlauf zum Auffammen gebracht werden können, was bei klinisch ähnlichen Intracutanquaddeln von Proteinkörpern nicht der Fall ist (v. Hayek, Bessau, eigene Beobachtungen). Weniger eindeutig stellt sich der Ablauf von Reaktionen im tuberkulösen Gewebe dar. Es scheint möglich, mit „unspezifischen“ Reizen der verschiedensten Art, vor allem mit Proteinen verschiedener Herkunft gleiche oder ähnliche Anfachungen des Entzündungsprozesses wie mit 'Tuberkulin zu erzielen (Albumosentheorie von Kühne, Buchner, Matthes, Krehl; Proteinkörpertherapie bei Tuberkulose nach $R$. Schmidt, Kraus, Kaznelson, A. Mayer u. a.). Die Autoren glaubten daher, die Spezifität der Tuberkuline ablehnen zu dürfen. Der Vorgang ist zu komplex, als daB man heute eindeutig entscheiden könnte, ob es sich bei der Tuberkulinreaktion um ein Spiel von Antigen und Antikörpern handelt (Wassermann und Bruck) oder wenigstens zum Teil -.. ob um eine reine Form der Anaphylaxie (Krehl) oder um die Wirkung eines Reizstoffes, der weder mit Antikörpern noeh mit Anaphylaxie etwas zu tun hat, sondern als Katalysator zu betrachten ist (Selter).

Es ist daher sehr wohl möglich, daß Reaktionen im tuberkulösen Gowebe qualitativ gleichartig ablaufen, ohne Rücksicht auf ihre kausale Genese. Die Vorstellung der Autotuberkulinisation $v$. Hayeks spricht vielleicht dafür. Es ist kaum anzunehmen, daß die Aktivierung oines Prozesses z. B. durch Masern oder Grippe ihrem Wesen nach von oinem Tuberkulinschadon verschieden sei.

Wonn Gegner der Spezifitit sie aus diesem Grunde ablehnen. wird man ihnen heute bei dem wenig befriedigenden Stand unserer Kenntnisse über den eigentlichen Charakter des Tuberkulins wenig widersprechen können. Aber selbt wenn sich jemand auf den Standpunkt einer rein anaphylaktischen Erklärung stellte, müßte man doch auch für das T.B.-Protein die auf diesem Gebiet gültigen Gesetze der strengen Spezifität gelten lassen. Verwandschafts- und Gruppenreaktionen lassen sich im Gebiet der ganzen Immunitätslehre aufweisen. Die Entscheidung wird gefällt durch die Dosen- 
menge, die zur Auslösung der Roaktion genügt. Ein krankes Organ, in dem sich chronische Entzïndungsprozesse abspielen, ist empfindlicher als gesundes Gewebe; es spricht auf viele Reize an. DaB es gegen einen besonderen Stoff stets besonders überempfindlich sei, macht die Beziehungen aus, die wir mit Spezifität bezeichnen.

In diesem Sinne darf das Tuberkulin als Stoff mit spezifischer Wirkung angesprochen werden. Es wirkt in kleinsten Dosen auf den tuberkulösen Organismus (7. B. MTbR.), in größten auf einen nicht infizierten nicht anders als Eiweißkörper in entsprechender Menge. Schon Mathes betonte die auffallende Potenz kleinster Tuberkulindosen und erklärte sie durch ein besonders giftiges Pepton. Selter sah beim Pepton bei intracutaner Einverleibung erst bei Mengen von $1 \mathrm{mg}$ Substanz eine deutliche Reaktion, bei TB bei $0.01 \mathrm{mg}$ und daruntel. Verfügen wir ïber einen derart wirksamen Stoff und wollen wir eine Reiztherapie in der Klinik der Tuberkulose anwenden, so scheint or von vornherein anderen, nicht so fein abgestimmten Reizstoffen überlegen - falls es uns nicht gerade auf eine allgemeine unspezifische Leistungssteigerung ankonmt (wio sie den Proteinkörpern seit Weichardt zugeschrieben wird), unter möglichster Ungehung der direkten Wirkung auf den Herd. Ob wir nun mit den Proteinkörpern die Möglichkeit in der Hand haben. empfindliche Herde stets schonend und milde zu beeinflussen oder ob ihre Wirkungsweise gelegentlich unberechenbar sein kann, bedarf jedenfalls eingehender Prüfung, da uns genügend brauchbare Tuberkuline verschiedener Reizstärke vorliegen.

Es muB daher Widorspruch hervorrufen, woun $R$. Schmidt seine Proteinkörpertherapie der Tuberkulose inauguriert mit den Worten: .Was die Tuborkulintherapie leistet, scheint die Milchtherapie auch zu leisten“. Der vorjährige Tuberkulosekongreß in Elster zeigte, wie übereinstimmend unsere namhaftesten Tuberkuloseforscher auf dem Boden der Spezifität stehen; die Frage ist, abgesehen von einer Diskussionsbemerkung Mayers kaum ventiliert worden. Und wenn zwar die einst hochgestimmten Erwartungen, dio sich an das Tuberkulin als immunisierendes Mittel knüpften, eine Ernüchterung erfahren haben, so scheinen die Ansichten über seine therapeutische Verwertbarkeit heute doch durchaus gleichmäßig gesicherte zu sein. Neufeld, Uhlenhuth u. a. vertraten die Ủberzeugung, daß die spezifische Therapie die im Körper vorhandenen Heilkräfte wirksam unterstützen kann. Kinstweilen scheint wenig Neigung, zugunsten der Proteinkörpertherapie in diesem ausschließlichen Sinne Schmidts und seiner Mitarbeiter das Feld zu räumen. Die Erfahrungen sind noch nicht ausreichend. Im vorigen Jahr berichtete Weicksel über unspezifisch behandelte Tuberkulosefälle und stellte die Tuberkulin- 
therapie über die unspezifischen, die er zu gefährlich fand. Caseosan soll danach leicht zu stark reizend wirken. Klemperer sah von Milchinjektionen keinen sicheren Nutzen und keinen Anlaß, sie an Stelle der spezifischen Therapie zu setzen.

Nachdem Sons und $v$. Mikulicz-Radecki an unserer Klinik die Frage der Spezifität der Tuberkuline im Vergleich ibrer Wirkungen mit der von Proteinkörpern auf den tuberkulösen Organismus experimentell in einer Peihe ron Tierversuchen und unter Benutzung der Intracutanreaktion am Menschen studiert hatten, schien es aussichtsreich, dieselbe Frage noch in anderer Beziehung zu verfolgen.

Die Intracutanreaktion, immer noch ein wenig geklärtes und viel umstrittenes Gebiet im Bereich der imnunbiologischen Zustandsänderungen, kann hier keinen vollbefriedigenden Aufschluß bringen. Boi der Frage nach der Wirkungsweise cines Reizstoffes (der Ausdruck sei als möglichst neutral und unverbindlich für das Tuberkulin wie für Proteine hier gebraucht) kommt es uns neben seiner Wirkung auf den tuberkulösen Gesamtorganismus vor allem auf die entzündlichen und hyperämischen Veränderungen an, die er im tuberkulösen Herd hervorruft, und ihre mittelbaren Folgen. Von diesem Herd ist ja jede Wirkung überhaupt erst abhängig. Neben einer Ergänzung der früheren Tierversuche soll der Zweck dieser Arbeit daher hauptsächlich sein, die Wirkung von Proteinkörpem auf Tuberkulöse mit den bekannten Reaktionen auf subcutane Tuberkulineinverleibung zu vergleichen. Um bei diesem Vorgehen nach Möglichkeit Schädigungen zu vormeiden, wurden die Injektionen des Eiweißkörpers (durchweg Caseosan) in den Rahmen einer Alttuberkulintherapie eingefügt, so daß unter diesen Bedingungen vielleicht gleichzeitig ein Urteil über die Möglichkeiten einer Proteinkörpertherapie bei Tuberkulösen gewonnen werden konnte. Davon wird im 2. Teil der Arbeit dio Rede sein.

\section{T. Versuche an Meerschweinchen.}

Als Sons unsere Klinik verließ, übernahm ich den Abschluß seiner Tierversuche und verfuhr zunächst ganz in seinem Sinne. Da der Proteingehalt der T.B. und der bekannten Tuberkuline den Grund für eine Identifizierung ihrer Reaktion mit jener der parenteral verabfolgten Proteinkörper bei chronischen Entzündungszuständen abgegeben hat, mußte jeder Vergleich ihrer Wirkungen von der Kenntnis ihres N-Gehalts ausgehen. Da die Konstanz dieses N-Gehalts weder bei Tuberkulinen noch bei den im Handel befindlichen Proteinkörpern eine gesicherte ist, überprüfte ich in einigen Kjeldahlbestimmungen, die hier wiedergegeben seien, die Sonsschen Zahlen (in Klammern): 
Es sind enthalten in

1 com Alttuberkulin (Stammlösung) 3?, $26 \mathrm{mg} \mathrm{N}(11,9 \mathrm{mg}$, dagegen Perlsucht 32,34)

1 , Aolan . . . . . . . $7,07, \mathrm{~N}(3,37)$

$1 "$ Caseosan . . . . . . . 6,79, $\mathrm{N}(5,67)$

1 "Abijon . . . . . . . . 5,39, $\mathbf{N}(-)$

Die Differenzen sind nicht unbeträchtlich; für Caseosan geringer als für das Alttuberkulin. Ich benutzte meine Werte, um den möglichen N-Gehalt des Tuberkulins eher zu hoch als zu niedrig anzusetzen ( $v$. Hayek schätzt nach Ruppel $1 \mathrm{ccm}$ AT auf $30 \mathrm{mg}$ Proteinsubstanzen). Das Tuberkulin ist in seiner Wirkung auf empfindliche tuberkulöse Herde in kleinsten Dosen dem Proteinkörper so weit überlegen, daß man bei diesem Vergleich den N-Gehalt des Tuberkulins getrost etwas zu seinem Nachteil berechnen darf, und wäre es, wio hier, vielleicht um das Dreifache. Es verhalten sich danach inbezug auf $\mathrm{N}$-Gehalt

$$
\begin{aligned}
\text { AT: Caseosan } & =4,7: 1 \\
(,: \text { Aolan } & =4,5: 1)
\end{aligned}
$$

oder eine bekannte Menge AT zur Errechnung des erforderlichen $\mathrm{N}$-Gehalts $\mathrm{x}$ einer Caseosandosis

$$
\text { AT: } x=1: 4,7 \text {. }
$$

Z. B.

$$
\begin{aligned}
0,3 \mathrm{ccm} \text { AT: } \mathrm{x} \text { Cas. }=1: 4,7 \\
\mathrm{x} \quad "=1,41 \mathrm{ccm} \text { Caseosan } \\
0,5 \text { AT: } \mathrm{x \quad} "=1: 4,7 \\
"=1,35 \mathrm{ccm} \text { Caseosan. }
\end{aligned}
$$

oder

\begin{tabular}{|c|c|c|c|c|}
\hline \multirow{6}{*}{ 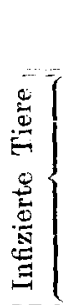 } & Nr. & $\begin{array}{l}\text { Dat um der } \\
\text { Injektion }\end{array}$ & $\begin{array}{l}\text { Menge und Stoff } \\
\text { intraperitoneal }\end{array}$ & Ergebnis \\
\hline & 32 & 2. VIII 21 & $\begin{array}{l}0,5 \mathrm{cem} \text { Alttuberkulin } \\
\text { (Stammlösung) }\end{array}$ & stirbt nach 8 Stunden \\
\hline & 45 & $"$ & $0,3 \mathrm{ccm}$ Alttuberkulin & 6 \\
\hline & 39 & $"$ & 2,5, Aolan & lebt bis Ende August \\
\hline & 42 & $n$ & $3,0 \Rightarrow$ Abijon & $" 31$. \\
\hline & 48 & $\eta$ & $-2,5 \quad$ Caseosan & $\approx \quad 1 \quad 25$. \\
\hline 0 & 29 & " & 0,5, Alttuberkulin & \\
\hline 定 & 28 & ” & $0,3 n \quad n$ & überleben sämtlich \\
\hline 足 & 56 & 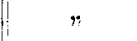 & 2,5, Aolan & bis Ende September \\
\hline छี & 26 & $"$ & 3,0 "Abijon & \\
\hline & 27 & $n$ & $2,5 \approx$ Caseosan & \\
\hline
\end{tabular}

Am 21. VI. 21 wurden 28 Meersehweinchen mit 1/, Öse Tb. 9 hum. (Kultur vom 14. VI. 21 Reichs-Ges.-A.) infiziert, subcutan linke Kniekehle. Von diesen Tieren starben vorzeitig, ehe sie für Versuchszwecke benutzt werden konnten, 21 von Ende Juni bis Mitte Juli an Stallseuchen. Die vergleichenden Untersuchungen an den überlebenden Tieren hatten das folgende Ergebnis: 
Am 24. VIII. 21 wurde der Versuch mit Tieren derselben Reihe wiederholt. Diosmal wurde 2 Tieren noch albumosefreies Tuberkulin injiziert, da Sons gefunden hatte, daß ein mit $0,5 \mathrm{ccm}$ A F. (ip) gespritztes Tier noch 31 Tage überlebte, und vermutete, das verwandte Tuberkulin könnte in seiner Wirkung unzuverlässig sein (Moro).

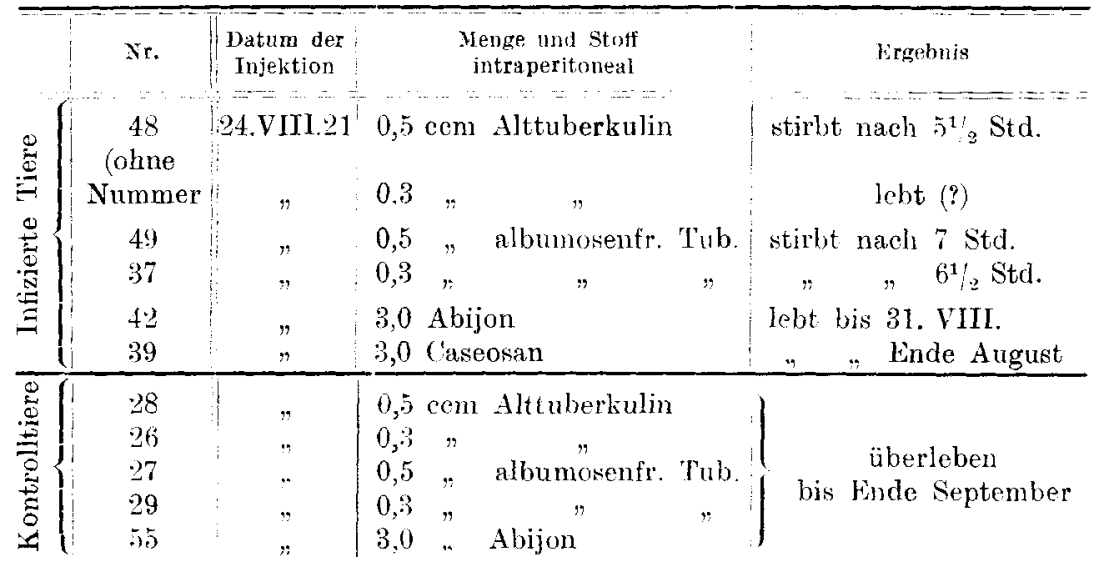

Bei allen infizierten Tieren wurde autoptisch, makroskopisch und mikroskopisch Tuberkulose nachgewiesen. Die tuberkulösen Veränderungen waren durchwog von ziemlich gleicher Ausdehnung. bei den Anfang August gestorbenen Tieren natürlich wesentlich geringer. Noben den linkseitigen Leistendrüsen waren am stärksten die Milz, danach die Leber erkrankt, die später eingegangenen Tiere wiesen außerdem sämtlich ausgedehnte Knötchen in den Lungen auf. Ủber das Schicksal des Tieres „ohne Nummer" ist mir leider nichts bekannt geworden. Der Todestag ist mir unbekannt geblieben, ebenso vermisse ich das Sektionsprotokoll. Der Ausfall ist daher nicht zu verwerten; möglichorweise handelte os sich überhaupt um ein Kontrollties.

Das Ergebnis der beiden Versuche ist augenfällig. Es handelt sich bei den Proteinkörperinjektionen um die gleiche bis doppelte Stickstoffmenge, als sich aus dem Tuberkulin berechnen läBt; trotzdem iiberleben alle mit ihnen gespritzten Tiere. Ich halte dabei in dem 2. Versuch für die Tiore $\mathrm{Nr} .42$ und 39 die Dauer von einel: Woche für ausreichend als Beweis; Ende August bostand die verhältnismäßig massige Infektion (1/, Ose) ïber 2 Monate und mußte auch spontan zum Tode führen.

Am 14. XII. 21 wurden 10 Tiere mit "/O Ose Tb. 1 (Stuttgart 17) hum. (Kultur vom 8. X. 21 Reichs-Ges.-Amt) in der gleichen Weiso infiziert. Von diesen Tieren starben vorzeitig 7 , durchweg an 
Lobulärpneumonien, eins an Pleuraempyem (im Ausstrich-Eiter keine Tbc.-B.). Es standen also nur 3 Tiere zur Verfügung. Am 26. I. 22 erhielt Tier Nr. 6 um $12^{30 \mathrm{~h}}$ 0,5 cem Alttuberkulinstammlösung intraperitoneal. Es starb nach 5 Stunden. 1/2 Stunde später wurdon die Tiere Nr. 7 und Nr. 10, die zur gleichen Zeit $\left(12^{30} \mathrm{~h}\right)$ $3,0 \mathrm{ccm}$ Caseosan bzw. $5 \mathrm{ccm}$ sterilisierte Kuhmilch intraperitoneal bekommen hatten, durch Schlag auf den Hinterkopf getötet. Sie waren vorher vollkommen munter und gaben keinerlei Krankheitszeichen zu erkennen, als Nr. 6 sich längst in der Agone befand. Soweit wäre der Versuch als eine gleichartigo Bestätigung der Vorversuche zu betrachten.

Zweck der Versuchsanordnung war nun außerdem, autoptisch und histologisch auf Veränderungen zu achten, die vielleicht als Herdreaktionen angesprochen werden könnten. Die Temperaturmessungen bei früheren Tierversuchen hatten uns keine eindeutigen Anhaltspunkte geben können, wann man die zeitliche Höhe einer Reaktion anzunehmen hat. Da die bisher mit Proteinkörpern gespritzten Tiere nie auf der Höhe einer Reaktion eingegangen waren, wurden Nr. 7 und 10 eben annähernd gleichzeitig mit dem Tod des AT.Tiers getötet.

Die Sektionsbefunde der Tiere sind nun folgende:

Nr. 6: In der Bauchhöhle etwas sanguinolente Flüssigkeit. Leistendrüsen links kleinerbsengroß, verkäst. Därme und Magen stark gebläht, intensiv gerötet. Dieselbe intensive Rötung zeigen alle Abdominalorgane (Leber, Milz, Nieren, Nebennieren). Besonders die Milz fällt durch ihre Größe und ihren Blutreichtum auf. In Leber und Milz zahlreiche Knötchen, Lunge makroskopisch frei. Hilusdrüsen etwas geschwollen, nicht vorkäst.

Nr. 7 : Leistendrüsen kleinerbsengroß, verkäst. Retroperitonealdrüsen vergrößert, verkäst. In der freien Bauchhöhle in Spuren etwas trüb seröse Flüssigkeit. Därme wenig aufgetrieben, blaß. Lober zeigt nur spärliche Tuberkel. Milz zahlreiche große Knötchen. Milz im ganzen kleiner als bei Nr. 6, auch kein auffallender Blutreichtum. Iungen klein, blasse Partien mit dunkelroten wechselnd (aspiriertes Blut). Nieren und Nebennieren o. B., kleiner als bei $\mathrm{Nr} .6$.

Nr. 10: Leistendrüsen links hohnengroli, verkäst. Retroperitonealdrüsen vergrößert, verkäst. Im Abdomen mehrere Kubikzentimeter milchiger Flüssigkeit. Leber makroskopisch keine sicheren Knötchen. Milz sehr klein, deutliche Knötchen. Abdominalorgane im ganzen eher blaß als blutreich. Langen wie bei Nr. 7.

Auch mikroskopisch fand sich in Schnitten von HämatoxylinEosin-Färbung dieser auffallende Cnterschied im Blutreichtum der tuberkulös erkrankten Organe bei den drei Tieren; eine starke Hyperämie in Umgebung der Herde von Tier Nr. 6. Vielleicht kann dieso Hyperämie als Herdreaktion angesprochen werden; immerhin könnte der Blutverlust der getöteten Tiere eine Rolle spielen. Das genaue Studium derartiger Veränderungen müßte natürlich eine größere 
Versuchsreihe umfassen und setzt pathologische Schulung voraus. Herr Dr. Siegel ging mir bei der histologischen Beurteilung der Präparate hilfreich zur Hand, wofür ihm an dieser Stelle gedankt sei.

\section{Subcutane Alttuberkulin- und Caseosaninjektionen bei tuberkulösen Menschen.}

Während diese Tierversuche, wie angenommen, in gleicher Weise ausfielen wie die von Sons und v. Mikulicz unternommenen und sie also weiter bestätigten, interessierte es mich vor allem, die Wirkung subcutan oder intramuskulär einverleibter Proteinkörper am tuberkulösen Menschen zu beobachten. Ist nach Schmidt das Tuberkulin in der Therapie Tuberkulöser entbehrlich? Lassen sich mit Eiweißstoffen unspezifischer Art (ich wählte hauptsächlich Caseosan) Herdund Allgemeinreaktionen nach Art der Tuberkulinreaktionen erzielen, und welche Mengen sind dazu erforderlich? Es mußten die Injektionen vergleichsweise an demselben Patienten durchgeführt werden, da die Empfindlichkeit Tuberkulöser gegen spezifische wie unspezifische Reize eine durchaus individuelle ist und Kranke mit klinisch ganz gleichem Befund in dieser Beziehung weit verschieden reagieren. Andrerseits ist aber auch an einem Individuum diese Empfindlichkeit keine konstante, sondern stetigen Verschiebungen, Entwicklungen nach der einen oder nach der anderen Seite unterworfen. Wer heute auf $1 \mathrm{mg} \mathrm{AT}$ z. B. reagiert, braucht es in 14 Tagen durchaus nicht mehr zu tun, und umgekehrt. So wird die nach dem N-Gehalt des Tuberkulins errechnete Caseosandosis nicht mehr die gleichen Verhältnisse antreffen, nachdem auf eine bestimmte Tuberkulingabe eine Reaktion erfolgte. Jede Reaktion muß das immunbiologische Zustandsbild verschieben, nach welcher Seite erkennen wir eigentlich erst aus der Antwort auf die nächste Injektion - sc. des gleichen, quantitativ und qualitativ genau bekannten Reizstoffes. Hier bleiben also 2 Unbekannte in der Rechnung (1. welche Caseosanmenge macht eine ähnliche 'Reaktion; 2. wie stark ist im Augenblick die Reaktionsempfindlichkeit des Herds?). Man uird die eine - nämlich die Frage der veränderten Reaktionsfähigkeit - notgedrungen vernachlässigen dürfen, wenn auf der andern Seite sich sinnfällige Unterschiede der Dosengröße erweisen lassen. Es mußte also möglichst gezeigt werden, daß nicht nur eine Dosis Caseoson vom gleichen N-Gehalt wie die entsprechende ATGabe dort keine Reaktion macht, wo diese AT-Gabe eine auslöste, sondern auch die doppelte, vielfache, ja eventuell 100-oder 1000 fache nicht.

Ich bin im allgemeinen so vorgegangen, daß ich in den Turnus einer ordnungsmäßigen Tuberkulintherapie dann eine Caseosaninjektion 
einschob, wenn die letzte AT-Menge eine deutliche Reaktion gemacht hatte. Dabei kam die Stichreaktion weniger in Frage; neben der Herdreaktion muBte die Allgemeinreaktion die wichtigere Komponente dieser Reaktion bilden --.. die Herdreaktion, weil die Frage nach der Spezifität eines Reizstoffes hauptsächlich durch die Wirkung auf den besonderen Krankheitsherd entschieden wird; die Allgemeinreaktion, weil sie unter. Umständen die objektivero sein kann. Unsere Fälle gehören durchweg zur Gruppe der tertiären Phthisen mit vorgeschrittenen Lungemprozessen. Die initialen Fälle lassen sich meistens nicht lange in der Klinik halten. Es sind also vorwiegend Patienten mit ausgedehnten Veränderungen, häufig mit Kavernenbildung, die ständig reichlich physikalisch nachweisbare Symptome aufweisen. Dabei hat die Zunahme von Rasselgeräuschen über Partien, wo schon vorher welche gehört wurden, nicht immer Beweiskräftiges und ist dem subjektiven Entscheid des Untersuchers mehr oder weniger unterworfen. Eine genaue, möglichst 4 malige tägliche Temperaturmessung bietet da häufig die bessere Handhabo für die Beurteilung der Reaktionen und die Leitung der Therapie.

Durch das Vorgehen der nachzeitigen Caseosaninjektion wird die Beurteilung der Wirksamkeit des Caseosans wahrscheinlich oft etwas zugunsten einer stärkeren Wirkung versehoben. Nach allgemeingültigen Anschauungen sind grob merkbare Tuberkulinreaktionen (deutliche Herd-, starke Allgemeinreaktion) nicht die besten, sondern stehen an der Grenze des Tuberkulinschadens. Bei einigen der im folgenden beschriebenen Reaktionen wird man zweifellos den Eindruck eines Schadens, d. h. einer Steigerung der Empfindlichkeit des Herdes mit Neigung zur Propagation gewinnen. Der dann einsetzende Reiz wird also häufig nur ein kleineser zu sein brauchen, um ähnliche Effekte zu erzielen. (Selbstverständlich ist bei mittleren Reaktionen ebensooft das Umgekehrte, die Resistenzsteigerung möglich. Sonst müßten wir ja immer in der Dosierung nach einel: Reaktion heruntergehen.)

Da mir die Wirkung des Caseosans auf Lungenherde durchaus unbekannt war, mußte die vertraute AT-Therapie als Maßstab dienen. Im Anfang wurden ganz kleine Mengen Caseosan (z. B. 0,003 ccm entsprechend $0,5 \mathrm{mg} \mathrm{AT}$ ) injiziert, nachdem ähnlich wie beim Tuberkulin Verdünnungen hergestellt waren. Narchdem sich dann zeigte, daß derartige Dosen wohl nie eine Reaktion hervorrufen können, blieben wir bei der Stammlösung und begnügten uns mit dem Herabgehen auf 2 bis 3 Strich. Schon diese Stellungnahme in der Mengenfrage beleuchtet deutlich die Úberlegenheit des Tuberkulins, so daß man dem Caseosan ruhig quasi den Vorsprung einräumen konnte, vielleicht öfters als das Tuberkulin einen empindlich gemachten 
Boden anzutreffen. Es konnte durch dieses Vorgehen unsere Frage ja nur sicherer entschieden werden.

Der zeitlichen Entstehung und Entwicklung der Versuchsbedingungen folgend -..-tastende evtl. sehr kleine Mengen von möglichst gleichem N-Gehalt im Beginn, später ${ }^{1}-3 \mathrm{ccm}$ Caseosan - sollen im folgenden die Versuchsprotokolle wiedergegeben werden. Zunächst soll der: Versuch gemacht werden, die Frage zu beantworten: lösen Caseosaninjektionen von glejchem N-Gehalt wie entsprechende ATDosen auch eine Reaktion aus, wemn die AT-Injektion dieses bewirkte? dann, da hiesauf durchweg eine verneinende Antwort erfolgte, möglichst die entsprechende Caseosanmenge gefunden werden, die dann imstande war, reaktiv zu wirken. Zuletzt soll ohne Anhaltspunkte für die Allergieverhältnisse des betreffenden Falles, wie sie durch eine Tuberkulinreaktion in positivem Sinne gegeben werden, in beliebigen Fällen stets eine relativ gleiche Caseosanmenge $(1-2 \mathrm{ccm})$ injiziert werden, um festzustellen, ob vielleicht diese Proteinkörpermenge konstant Reaktionen macht, unabhängig von immunbiologischen Verhältnissen. Eine scharfe Trennung in 3 Gruppen wird sich dabei nicht durchführen lassen, immerhin soll möglichst nach dieser Disposition vorgegangen werden.

Fall Nr. 1: La., Friedrich, 39jähriger Arleiter. 14. IX. 21, hei AbschluB der Arbeit noch in stationärer Behandlung.

Diagn.: Proliterierend-indurrierende I'uberkulose beider Oberlappen.

Hereditär o. B. Seit 1916 Jungenkrank, öfters Hämoptoe. - MäBiger Ernährungs- und Kräftezustand. Durchweg afebril. Ausgedehnte Prozesse in beiden Oberlappen, geringere in den Lnterlappen, Mischung produktiver Herde mit alten cirrhotischen Prozessen. Verdacht auf Cavum r. o. Pleuraschwarte l., Verziehung des Herzens nach I. Sputummenge: zwischen 50 und $100 \mathrm{ccm}$ in 24 Std., T.B. + . Immunbiologisch; allergisch mit geringer Tendenz zur positiven Anergie. (Wir verwenden die Ausdrücke positive und negative Anergie in Sinne v. Hayeks).

$$
\begin{gathered}
\text { Gewicht bei Aufnahne } 63,5 \mathrm{~kg} \text {, } \\
\text { Ende März } 68,3 \% \text {, } \\
\text { häufig sehwankend. }
\end{gathered}
$$

Therapie: AT, Beginn mit $0,001 \mathrm{mg}$, bisher bis $40 \mathrm{mg}$.

\begin{tabular}{|c|c|c|c|c|c|c|c|}
\hline tnjektion von & $\begin{array}{c}\text { Datnm } \\
14: 1 \\
\end{array}$ & $\begin{array}{l}\text { Rekt } \\
\mathrm{s}^{\mathrm{b}}\end{array}$ & ale Ten & $4^{\text {h }}$ & $s^{b}$ & $\begin{array}{l}\text { Menge des } \\
\text { Sputums } \\
\text { in erm }\end{array}$ & Bemerk ungen \\
\hline $0,2 \mathrm{mg} \mathrm{AT}$ & $\begin{array}{l}28 . \mathrm{X} \\
31 . \mathrm{X}\end{array}$ & & $\begin{array}{l}\text { nor } \\
37,1\end{array}$ & $\begin{array}{l}\mathrm{mal} \\
37,4\end{array}$ & 37,8 & $\begin{array}{l}50 \\
70\end{array}$ & realiti \\
\hline $0, \overline{5} \mathrm{mg} \mathbf{A T}$ & $\begin{array}{c}\text { 1. XI. } \\
\text { 2. XI. } \\
\text { 3. XI. } \\
\text { 4. -6. XI. }\end{array}$ & $\begin{array}{l}36,5 \\
\mathbf{3 9 , 0} \\
36,8\end{array}$ & $\begin{array}{r}36,8 \\
\mathbf{3 8 , 4} \\
37,0 \\
\text { nor }\end{array}$ & $\begin{array}{l}37,3 \\
\mathbf{3 8 , 1} \\
37,0 \\
\text { mal }\end{array}$ & $\begin{array}{l}40,0 \\
38,0 \\
37,2\end{array}$ & $\begin{array}{r}100 \\
50 \\
50 \\
50\end{array}$ & $\begin{array}{r}\text { Exbrechen StR } \theta \\
\mathbf{H R}-\end{array}$ \\
\hline
\end{tabular}

Schon bei den Anfangsdosen häutig leichte Temperatursteigerungen, keine deutlichen Herdreaktionen. 
(Fortsetzung.)

\begin{tabular}{|c|c|c|c|c|c|c|c|}
\hline \multirow{2}{*}{ Injektion von } & \multirow{2}{*}{$\begin{array}{l}\text { Oatum } \\
1921\end{array}$} & \multicolumn{4}{|c|}{ Rektale Temperaturen ${ }^{0}$} & \multirow{2}{*}{$\begin{array}{l}\text { Menge des } \\
\text { Sputums } \\
\text { in ccm }\end{array}$} & \multirow{2}{*}{ Bemerkungen } \\
\hline & & $8^{4}$ & $12^{\mathrm{h}}$ & $4^{31}$ & $8^{\mathrm{h}}$ & & \\
\hline \multirow{3}{*}{$\begin{array}{l}0,003 \text { cem Case- } \\
\text { osan }\end{array}$} & 7. XI. & 36,4 & 36,5 & 36,6 & 36,0 & 50 & $2 \mathrm{St}$ auf; Höhen- \\
\hline & 8. XI. & 36,6 & 37,0 & 36,9 & 37,2 & 40 & $\operatorname{StR} \theta$ \\
\hline & 9. XI. & 36,3 & 36,6 & 36,4 & 37,2 & 50 & HR $\theta$ \\
\hline \multirow[t]{4}{*}{$0,005 \mathrm{cem}$ Cas. } & 10. XI. & 36,2 & 36,6 & 37,1 & 37,1 & 50 & StR $\theta$ \\
\hline & 11. XI. & 36,3 & 37,6 & 37,0 & 37,2 & 50 & $\mathrm{HR} \theta$ \\
\hline & 12. XI. & 36,5 & 36,3 & 36,8 & 36,8 & 50 & Bettruhe \\
\hline & $13 . n .14 . X I$ & & & mal & & 50 & \\
\hline \multirow[t]{6}{*}{$0,5 \mathrm{mg} A \mathrm{~T}$} & 15. XI. & 36,8 & 37,0 & 37,0 & 37,4 & 50 & $\mathrm{StR}$ \\
\hline & 16. XI. & 36,0 & 37,4 & 37,7 & 37,7 & 50 & $\mathrm{HR}\}$ \\
\hline & 17. XI. & $\mathbf{3 1 , 1}$ & 37,0 & 37,0 & 37,5 & 50 & \\
\hline & 18. XI. & 36,6 & 37,2 & 38,0 & 37,6 & 50 & \\
\hline & 19. XI. & & & $\mathrm{mal}$ & & $50-$ & \\
\hline & 20.X1. & & no & mal & 37,8 & 100 & 4 Std. auf \\
\hline \multirow[t]{3}{*}{0,05 cem Cas. } & 24. XI. & 36,4 & 36,7 & 37,1 & 37,4 & 50 & $\operatorname{StR})_{\theta}$ \\
\hline & 25. XI. & 36,6 & 37,4 & 36,8 & 37,4 & 50 & HR $f$ \\
\hline & 26.XI. & 36,4 & 37,2 & 37,4 & 37,5 & 50 & lraine Pealtion \\
\hline $\mathrm{U}, \mathrm{O} \mathrm{mg} \mathrm{AT}$ & $29 . X 1$. & & & mal & & & keine Reaktion \\
\hline
\end{tabular}

Bemerkung: StR $=$ Stichreaktion: $\theta=$ nicht nachweishar:

HR $:=$ Herdreaktion; $+=$ vorhanden.

Dgl. im wesentlichen bis $5 \mathrm{mg}$; hierbei Allgemeinreaktion bis $38^{\circ}$, Stichreaktion, Husten vermehrt, Auswurfmenge steigt auf $200 \mathrm{ccm}$. Physikalisch keine sichere Anderung. Dann bis $: 0 \mathrm{mg}$ gut vertragen.

\begin{tabular}{|c|c|c|c|c|c|c|c|}
\hline \multirow{2}{*}{ Injektion vorl } & \multirow{2}{*}{$\begin{array}{c}\text { I) at um } \\
1922 \\
\end{array}$} & \multicolumn{4}{|c|}{ Rektale Temperaturen } & \multirow{2}{*}{$\begin{array}{l}\text { Menge des } \\
\text { Spntums } \\
\text { in ccm }\end{array}$} & \multirow{2}{*}{ Bemerkungen } \\
\hline & & $8^{3}$ & $1 y^{\mathrm{b}}$ & $t^{n}$ & $8^{6}$ & & \\
\hline \multirow{5}{*}{$40 \mathrm{mg}$ AT } & 13. JII. & 36,6 & 36,8 & 37,1 & 37,8 & 100 & \multirow{5}{*}{$\begin{array}{l}\text { StR } \\
\text { HR }\end{array}$} \\
\hline & 14. III. & 36,4 & 37,0 & 37,1 & 38,5 & 250 & \\
\hline & 15. III. & 38,9 & 37,4 & 36,8 & 37,3 & 100 & \\
\hline & 16. III. & 36,4 & 37,2 & 37,5 & 37,1 & 100 & \\
\hline & $17 .-20$. III. & & nor & mal & & 50 & \\
\hline \multirow[t]{3}{*}{$0,5 \mathrm{cem}$ Cas. } & 21. III. & 36,4 & 37,0 & 36,5 & 37,0 & 40 & \multirow{3}{*}{$\left.\begin{array}{l}\text { StR } \\
\text { HR }\end{array}\right\}$} \\
\hline & 22. III. & 86,1 & 37,0 & 36,8 & 37,2 & 70 & \\
\hline & 23. III. & 36,6 & 37,0 & 37,3 & 37,2 & 100 & \\
\hline \multirow[t]{4}{*}{$1,0 \mathrm{ccm}$ Cas. } & 24. III. & 36,4 & 37,3 & 37,2 & 37,6 & 90 & \multirow{4}{*}{$\begin{array}{l}\text { StR } \ldots \\
\text { HR } \theta\end{array}$} \\
\hline & 25. UII. & 36,0 & 37,0 & 37,0 & 36,8 & 90 & \\
\hline & 26. ПII. & 36,2 & 37,0 & 37,2 & 37,0 & 60 & \\
\hline & 27. III. & 36,6 & 37,2 & 36,9 & 36,9 & 50 & \\
\hline \multirow[t]{3}{*}{$40 \mathrm{mg} \mathrm{AT}$} & 28. III. & 36,3 & 36,6 & 37,2 & 39,0 & 100 & \multirow{3}{*}{$\begin{array}{l}\text { StR } \\
\text { HR :- } \\
\text { (mehr Husten) } \\
\text { vorne Rg }\end{array}$} \\
\hline & 29. III. & 37,6 & 37,7 & 37,7 & 37,5 & 150 & \\
\hline & 30. III. & 36.7 & 36,7 & & & & \\
\hline
\end{tabular}


Der Fall zeigt folgendes:

Es rerhalten sich $0.5 \mathrm{mg} \mathrm{AT}: \mathrm{x}$ Cas. $=1: 4,7$

$$
\begin{aligned}
\mathrm{x} & =2,35 \mathrm{mg} \\
& =0,01235 \mathrm{ccm} \text { Cas. }
\end{aligned}
$$

Während also $0, \check{c} \mathrm{mg} \mathrm{AT}$ am 1 . XI. eine starke Allgemeinreaktion und eine deutliche Herdreaktion auslöst, wird die entsprechende Menge Caseosan $=0,003 \mathrm{ccm}$ reaktionslos vertragen, ebenso am 10. XI. die doppelte Menge von $0,005 \mathrm{ccm}$. Am 15. XI. auf $0,5 \mathrm{mg}$ AT eine fragliche Allgemeinreaktion; $0,05 \mathrm{ccm}$ Caseosan - = 20 fache $N$-Menge keine Reaktion. Dasselbe Verhalten läßt sich im März bei $40 \mathrm{mg}$ A $\mathrm{T}$ noch einmal zeigen:

$$
\begin{aligned}
40 \mathrm{mg} \text { AT: }: \text { Cas. } & =1: 4,7 \\
x & =188 \mathrm{mg} \\
& =0,2 \mathrm{ccm} \text { Cas. }
\end{aligned}
$$

$40 \mathrm{mg}$ A T ruft wieder deutliche Allgemein- und Herdreaktion hervor: $0,5 \mathrm{ccm}$ und $1,0 \mathrm{ccm}$ Caseosan keine Reaktion; am 28. März auf $40 \mathrm{mg}$ AT wieder starke Allgemein-, deutliche Herdreaktion, als Zeichen, daB die Herdempfindlichkeit inzwischen nicht abgenommen hat.

Fall Nr. 2: Pat., Hermann, 18 jähriger Kinecht.

18. V. bis 18. VI. 1921 und 30. VT 1921 bis 27.1 . 1922.

Diagnose: Proliferierend-indurierende T'bc. beider Oberlappen $l>r$. Hereditär belastet (Vater an Tbc. gestorben, Mutter krank). Seit November 1920 suspekte Anamnese. -- Guter Allgemeinzustand; subfebril. Klinisch und röntgenologisch produktive Herde mit cirrhotischen Veränderungen gemischt im Bereich des linken Oberlappens, kleine Pleuraadhäsion 1.; geringere Veränderungen im mittleren Drittel des r. Oberlappens. Sputummenge etwa $50 \mathrm{ccm}$; T.B. + Immunbiologisch: schwach allergisch, Neigung zu positiver Anergie. Bei Entlassung Sputum T.B. $\theta$.

$$
\begin{aligned}
& \text { Gewicht bei Aufnahme } 49,4 \mathrm{~kg} \text {; } \\
& \text { bei Entlassung } 55,0 \mathrm{~kg} \text { (Heilstätte). }
\end{aligned}
$$

Therapie: AT, Beginn mit $0,01 \mathrm{mg}$, durchgeführt bis $60 \mathrm{mg}$. Bis $40 \mathrm{mg}$ keine stärkere Reaktion, leichte Temperatur-Steigerungen im Verlauf der Injektionstherapie bisweilen, ebenso Stichreaktionen.

Nach mehrtägiger fieberfreier Vorperiode (s. folgende Tabelle)

Es scheint mir zweifolhaft, ob man die Temperatursteigerung auf $38,0^{\circ}$ an 4. Tag noch als Allgemeinreaktion auf das Caseosan auffassen darf, viel eher auf Aufstehen und Höhensonne. Jedenfalls läBt sie sich mit dem Fieber am 25. und 26. X. nicht vergleichen; damals Herd- und Stichreaktion, beide fehlen jetzt. Die Menge von $0,3 \mathrm{ccm}$ Caseosan entspricht in N-Gehalt ziemlich genau einer Dosis von $60 \mathrm{mg}$ AT:

$$
\begin{array}{rl}
60: \mathrm{x}: & 1: 4, \overline{7} \\
\mathrm{x} & 282 \mathrm{mg} \\
- & 0,3 \mathrm{ccm} \text { Caseosan. }
\end{array}
$$




\begin{tabular}{|c|c|c|c|c|c|c|c|}
\hline \multirow{2}{*}{ Injektion vou } & Datum & \multicolumn{4}{|c|}{ Rektale Temperaturer ${ }^{\circ}$} & Menge d. & \multirow{2}{*}{ Bemerkungen } \\
\hline & 1921 & $8^{\mathfrak{b}}$ & $19^{\mathrm{h}}$ & $4^{\text {h }}$ & $8^{\mathrm{b}}$ & in $\mathrm{cm}$ & \\
\hline \multirow{6}{*}{$60 \mathrm{mg}$ AT } & $23 . \mathrm{X}$. & $: 86,7$ & 37,4 & 37,5 & 37,5 & 50 & \multirow{9}{*}{$\begin{array}{l}\text { StR } \\
\mathbf{H R}\end{array}$} \\
\hline & 24. X. & 36,65 & 37,3 & 37,5 & 37,5 & 50 & \\
\hline & $25 . \mathrm{X}$ & 36,4 & 37,3 & 37,2 & 39,8 & 90 & \\
\hline & 26. X. & 39,5 & 38,8 & 38,3 & 38,4 & 100 & \\
\hline & 27. $X$. & 37,1 & 37,3 & 37,0 & 37,5 & 150 & \\
\hline & $28 . \mathrm{X}$ & 36,8 & 37,2 & $: 37,1$ & 37,3 & 70 & \\
\hline \multirow{7}{*}{$0,3 \mathrm{cem}$ fas } & $29 . x$ & 36,1 & 37,5 & 37,5 & 37,5 & 40 & \\
\hline & $30 . x$ & 36,6 & 36,7 & 37,3 & 37,9 & 50 & \\
\hline & $1 . b .4 . \mathrm{XI}$ & \multicolumn{4}{|c|}{ normal } & 150 & \\
\hline & 5. XI. & 36,3 & 37,5 & 37,0 & $37, \overline{0}$ & 100 & str \\
\hline & 6. XI. & 36,3 & $: 37,4$ & $3 \pi, 4$ & 37,4 & 100 & HR $\}^{\theta}$ \\
\hline & i1 $7 . \mathrm{XI}$. & 36,4 & 37,4 & 37,6 & 37,6 & 140 & Aufstehen \\
\hline & ji $\otimes . X I$. & 36,3 & 37,3 & $\because 7,4$ & 38,0 & 100 & Höhensonne \\
\hline
\end{tabular}

Es folgt jetzt wieder eine fieberfreie Periode bis zum 17. XI. unter symptomatischer Therapie.

\begin{tabular}{|c|c|c|c|c|c|c|c|}
\hline \multirow{2}{*}{ Injektion von } & \multirow{2}{*}{$\begin{array}{c}\text { Datum } \\
1921\end{array}$} & \multicolumn{4}{|c|}{ Rektale Temperatıren o } & \multirow{2}{*}{$\begin{array}{l}\text { Menge des } \\
\text { Sputums } \\
\text { in ccm }\end{array}$} & \multirow{2}{*}{ Bemerkungen } \\
\hline & & $8^{4}$ & $12^{\mathrm{h}}$ & $4^{\mathrm{ln}}$ & $8^{\mathrm{h}}$ & & \\
\hline \multirow{6}{*}{$60 \mathrm{mg} / \mathrm{T}$} & 36. XI. & 36,4 & 37,2 & 37,2 & 37,3 & 60 & \multirow{6}{*}{$\begin{array}{l}\text { Höhensonne } \\
\text { StR } \theta \\
\text { HR ? }\end{array}$} \\
\hline & 17. XI. & 36,3 & 37,0 & 37,1 & 37,4 & 50 & \\
\hline & 18. XI. & 36,4 & 37,5 & 39,2 & 40,5 & 100 & \\
\hline & 19. XI. & 39,2 & 38,0 & 36,4 & 36,8 & 100 & \\
\hline & 20. XI. & 37,4 & 37,8 & 37,5 & 37,3 & 120 & \\
\hline & 21. XI. & 36,5 & 36,9 & 37,0 & 37,4 & 100 & \\
\hline
\end{tabular}

Patient klagt am 18. und 19. über stärkeren Husten (und Auswurf). Obj. Rasselgeräusche gleich. Keine Reaktion der Einstichstelle, dagegen Auflammen alter Intracutanquaddeln vom 7. XI.

$$
\begin{array}{cc}
\text { (AT 0,1 cem Lsg. } 1: 1 \text { Million } \\
\text { dgl. } & 1: 100000 \\
\text { dgl. } & 1: 10000) .
\end{array}
$$

Die Caseosaneinstichstelle ist nicht mehr aufzufinden. Nachdem Patient sich wieder im alten fieberfreien Zustand befindet, die Auswurfmenge auf $50 \mathrm{ccm}$ zurückgegangen ist, erfolgt an 6. XII. eine Injektion von $0,5 \mathrm{ccm}$ Caseosan (s. folgende Tabelle):

Der Fall zeigt also bei $60 \mathrm{mg} \mathrm{AT}$, anscheinend seiner derzeitigen Grenzdosis, konstant eine äußerst kräftige, prompt einsetzende Reaktion, während die eingeschaltete Dosis Caseosan von gleichem N-Gehalt keine, die folgenden 0,5 und $0,7 \mathrm{ccm}$ (also fast bzw. mehr als doppelte N-Menge) nur schwache, verschleppte Reaktionen auslösen, jedenfalls keine merkliche Herdreaktion. Die Temperaturen des 25. XII. sind noch angeführt, um zu zeigen, daß derartige Temperatur- 
Die Spezifität der Tuberkulinreaktion.

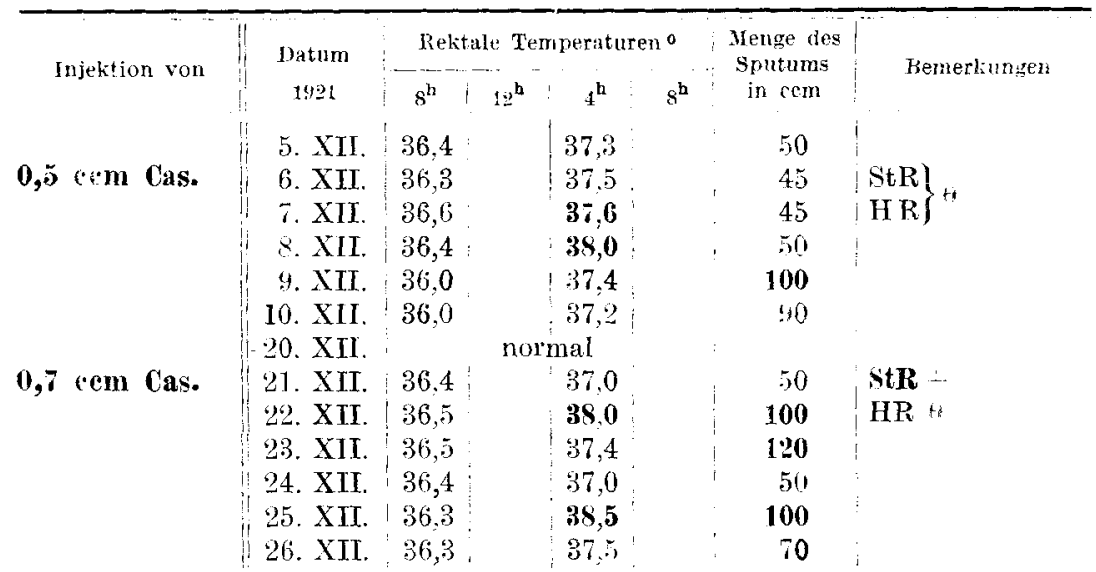

steigerungen, wie (aseosan sie hervorrief (?), auch ohne deutlichen Grund, durch jeden andern unspezifischen Reiz bei diesem Fall auftreten konnten.

Fall Ar. 3: Ra., Lisbeth, 16 jährige Haustochter. 8. VI bis 8. XII. 1921.

Diagnose: Proliftrierend-indutierende Tbe beider Oberlappen $7>r$. Larynx-Tbc. Erbliche Belastung fraglich. Fräher nie krank. Seit November 1918 (nach Grippe Tbc.-suspekte Anamnese. - Guter Emährungszustand. Nach Periode subfebriler Temperaturen im wesentliehen afebriler Verlauf. Klinisch und röntgenologisch vorwiegend proliferierende Spitzenherde, geringere in der Hilusgegend $1 .>$ r. Initialer spezifischer Larynxprozeb. Sputummenge wechselnd, 60 und weniger ccm, T. B. †. Immunbiologisch: stark allergisch. Unter vorsichtiger AT. und Krysolganbehandlung Besserung des Iungen- und Kehlkopfbefundes.

Gewicht bei Aufnahme $57,6 \mathrm{~kg}$, bei Entlassung $60,5 \mathrm{~kg}$ (Heilstätte).

Therapie: AT, Beginn mit $0,001 \mathrm{mg}$, durchgeführt bis $5 \mathrm{mg}$. Auf relativ geringe Dosen oft Temperatursteigerung und Herdreaktion (bei $0,07 \mathrm{mg} \mathrm{z}$. B. bis $\left.39,0^{\circ}\right)$. Zuletzt $2 \mathrm{mg}$ gut vertragen.

\begin{tabular}{|c|c|c|c|c|c|c|c|}
\hline \multirow{2}{*}{ Injektion vol } & \multirow{2}{*}{$\begin{array}{c}\text { Watum } \\
1921\end{array}$} & \multicolumn{4}{|c|}{ Rektale Temperaturen ${ }^{\circ}$} & \multirow{2}{*}{$\begin{array}{l}\text { Menge des } \\
\text { Sputums } \\
\text { in cem }\end{array}$} & \multirow{2}{*}{ Bernerkungen } \\
\hline & & $8^{h}$ & $12^{\mathrm{h}}$ & $4^{b}$ & $8^{h}$ & & \\
\hline & $27 . X$. & 36,7 & 37,4 & 37,0 & 37,1 & 40 & \\
\hline \multirow[t]{4}{*}{$3 \mathrm{mg}$ AT } & $28 . \mathrm{X}$ & 36,7 & 37,2 & 37,3 & 37,2 & 50 & $\mathrm{StK}$ \\
\hline & $29 . \mathrm{X}$. & 37,4 & 38,1 & 38,2 & 37,5 & 40 & HR \\
\hline & 30. X. & 36,5 & 36,4 & 37.2 & 37.1 & 50 & \\
\hline & $31 . \mathrm{X}$. & 36,8 & 37,0 & 37,0 & 36,8 & 50 & ¡ \\
\hline \multirow[t]{4}{*}{$0,02 \mathrm{cem}$ Cas. } & $1 . X I$. & 36,6 & 37,2 & 37,2 & 36,5 & 40 & StR \\
\hline & $2 . \mathrm{XI}$ & 36,5 & 36,9 & 37,1 & 36.7 & 40 & $0.2 \mathrm{Kry}-$ \\
\hline & 3.XI. & 36,7 & 37,1 & 37,1 & 36,7 & 30 & solgan. \\
\hline & 4.XI. & 36,6 & 37,1 & 37,3 & 36,4 & 30 & ; \\
\hline \multirow[t]{3}{*}{0,2 com cas. } & $5 . \mathrm{XI}$ & 36,5 & 37,0 & 37,0 & 36,7 & 40 & StR: \\
\hline & 6. XI. & 36,7 & 37,2 & 37,4 & 37,0 & 20 & $\mathrm{HR}$ \\
\hline & b.10.XI. & & no & mal & & & \\
\hline \multirow[t]{3}{*}{$3 \operatorname{nig} \perp \mathrm{T}$} & 11. XI. & 36,5 & 37,4 & 37,2 & 37,0 & 30 & $\mathrm{StR}-$ \\
\hline & $12 . \mathrm{XI}$ & 37,2 & 37,9 & 38,0 & 37,8 & 30 & HR $\theta$ Brust. \\
\hline & 13.XI & 36.7 & 37,8 & 37,2 & 36,8 & $\because 0$ & schmerzen) \\
\hline
\end{tabular}


Einer Menge von $3 \mathrm{mg}$ AT würde in bezug auf den N-Gehalt: $0,01 \pm \mathrm{ccm}$ Casoosan entsprechen. Für $3 \mathrm{mg}$ AT besteht eine ziemlich konstante Empfindlichkeit (am 25. XI. erfolgt auf $3 \mathrm{mg}$ eine ganz ähnliche Reaktion $\left[38,0^{\circ}\right]$, erst am 28. roaktionslos vertragen: am 1. XII. auf $3 \mathrm{mg} 37,9^{\circ}$ am 5. XII. auf 5 in $38,1^{\circ}$ und deutliche Herdrektion), während sogar die 14 fache $\mathrm{N}$-Menge in $0,2 \mathrm{~cm}$ Caseosan unter sonst gloichen Verhältnissen reaktionslos vertragen wird (ganz geringe Stichreaktion).

Fall Ni. 4: Ki., Frieda, 22 jährige Haustochter. 8. VI bis 19. XII. 1!21. Diagnose: Vorwiegend induricrende Tbc. ler linken Lunge. Vater an Lungenentzündung gestorben, eine Schwester an Himhautentzündung. Soit September 191 Tbc-Anamnese. - MäBiger Ernährungs- und Kräftezustand; durchweg afehril. Ausgedehnter, rorwiegend cirrhotischer Prozeß der linken Iunge mit Pleuraschwarte und großr Caverne im Oberlappen. Linke Thoraxseite geschmumpt. Chronische Pharyngitis. Sputummenge: etwa $10-40 \mathrm{cem}$ durchschnittlich, T.B. '. Immunhiologisch: Neigung zu positiver Anergie

Gewicht bei Aufnahme $57,3 \mathrm{~kg}$, .

bei Entlassung $61,3 \mathrm{~kg}$ (Heilstätte.

Stellt sich Mitte März 1922 mit $10 \mathrm{~kg}$ Gewichtszunahme und ganz geringem katarrhalischen Befund nach Rückkehr aus der Heilstätte vor.)

Themnie: AT, Beginn mit 0,05 $\mathrm{mg}$, daun 0,1; durchgeführt bis $50 \mathrm{mg}$.

Bis $30 \mathrm{mg}$ heine wesentliche Reaktion, Temperaturen norual, geringe Stich-, keine Herdreaktion.

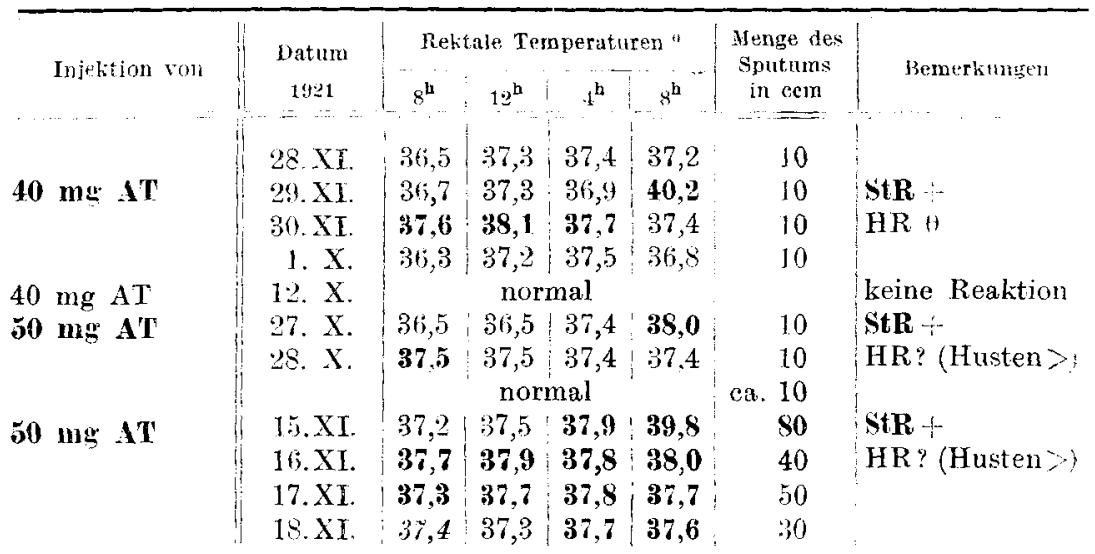

Es folgt nach einigen fast fieberfreien Tagen vom 22. bis 30 . XI. eine Periode febriler Temperaturen mit vermehrtem Auswurf, frischem pleuritischen Reiben und Brustschmerzen. Anscheinend bedeuten $50 \mathrm{mg}$ die derzeitige Grenzdosis, vielleicht ist auch eine Aktivierung des Prozesses durch die letzte Injektion anzunehmen. Dieses Stadium mußte erst abgewartet werden, ehe vergleichsweise 
Caseosan injiziert werden konnte. Man mußte erwarten, die Krankheitsherde gegen jegliche Reize empfindlicher zu finden. Ich injizierte am 6. XIr. 0,5 ccm Caseosan (50): $\quad 1: 4,7: x: 235 \mathrm{mg}=0.235 \mathrm{ccm}$ ), also die doppelte $\mathrm{N}$-Monge in Vergleich zu $50 \mathrm{mg}$ AT.

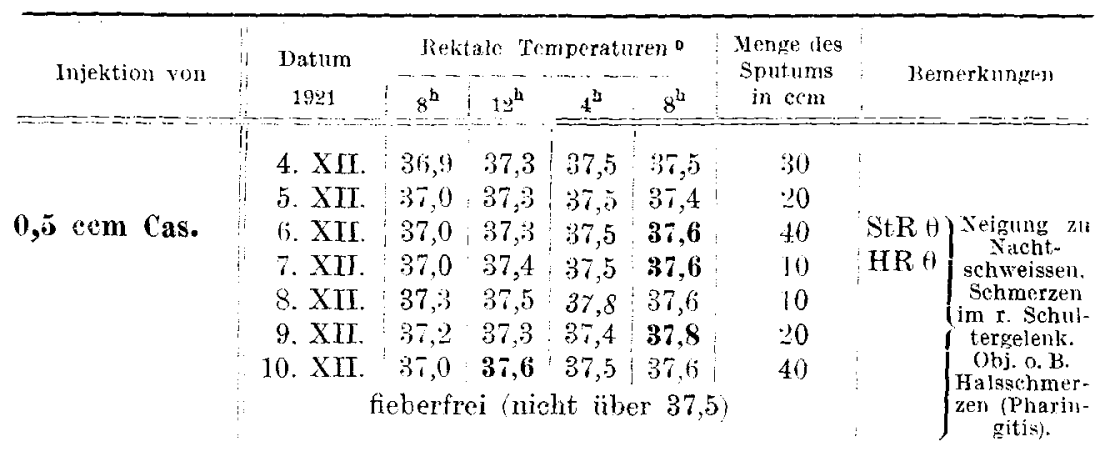

Pat. fühlt sich wieder wohler, hat auch am 6. XII. und am 13. XII wieder: an Gewicht zugenommen, das unter den letzten ATDosen zurückgegangen war. Der Tukerkulinschaden (?) scheint überwunden.

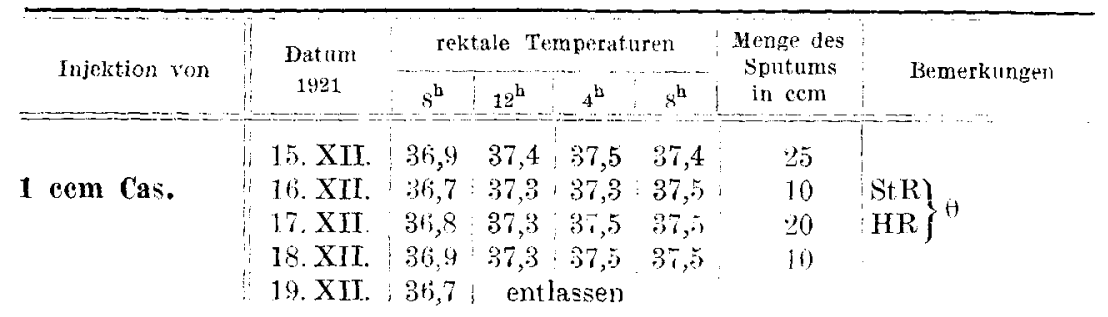

1 com Caseosan enthält über dje +fache N-Menge von $\overline{0}(\mathbf{m g}$ $\mathrm{AT}$; es wird olne jede Reaktion vertragen. Die Injektion ron $0,5 \mathrm{ccm}$ am 6 . XII. trifft wahrscheinlich einen weniger widerstandsfähigen Organismus. Eine sichere Reaktion ist auch hierbei nicht zu verzeichnen; wobei unentschieden gelassen werden muss, ob Schulter- und Halsaffektion als Ursache der geringen Temperatursteigerungen anzusprechen sind oder als Begleit- und Folgeerscheinung einer verschleppten Allgemeinreaktion durch Caseosan:

Fall Nr. 5: K1., Hoinrich, 32-jähriger Arbeiter, 28. X. 1921 bis 8. II. 1992.

Diagnose: Proliferierend-indurierende $T b c$ beider oberlappen $r>l$. Vater und Schwester an Tbe gestorben. Schon als Kind Neigung zu Husten und Erkältungen. Seit September 1921 deutliche Tbc-Anamnese.-Guter Ernährungszustand. Im Beginn subfebril, später durchweg afebril. Klinisch und röntgenologisch vorwiegend idurierende Prozesse der $r$. Lunge. Pleuraschwarte 
und Kaverne r. o. Sputummenge zwischen 50 und $100 \mathrm{com}$, T. B. ... Immunbiologisch Neigung zu positiver Anergie. Anfangs geringer Katarrh. der im Verlauf der Behandlung verschwindet.

Gewicht bei Aufnahme $61,4 \mathrm{~kg}$.

bei Entlussung $6: 5,7 \mathrm{~kg}$.

Therapie: AT, Beginn mit 0,01 $\mathrm{mg}$, ambulant bisher lis $1 \mathrm{mg}$.

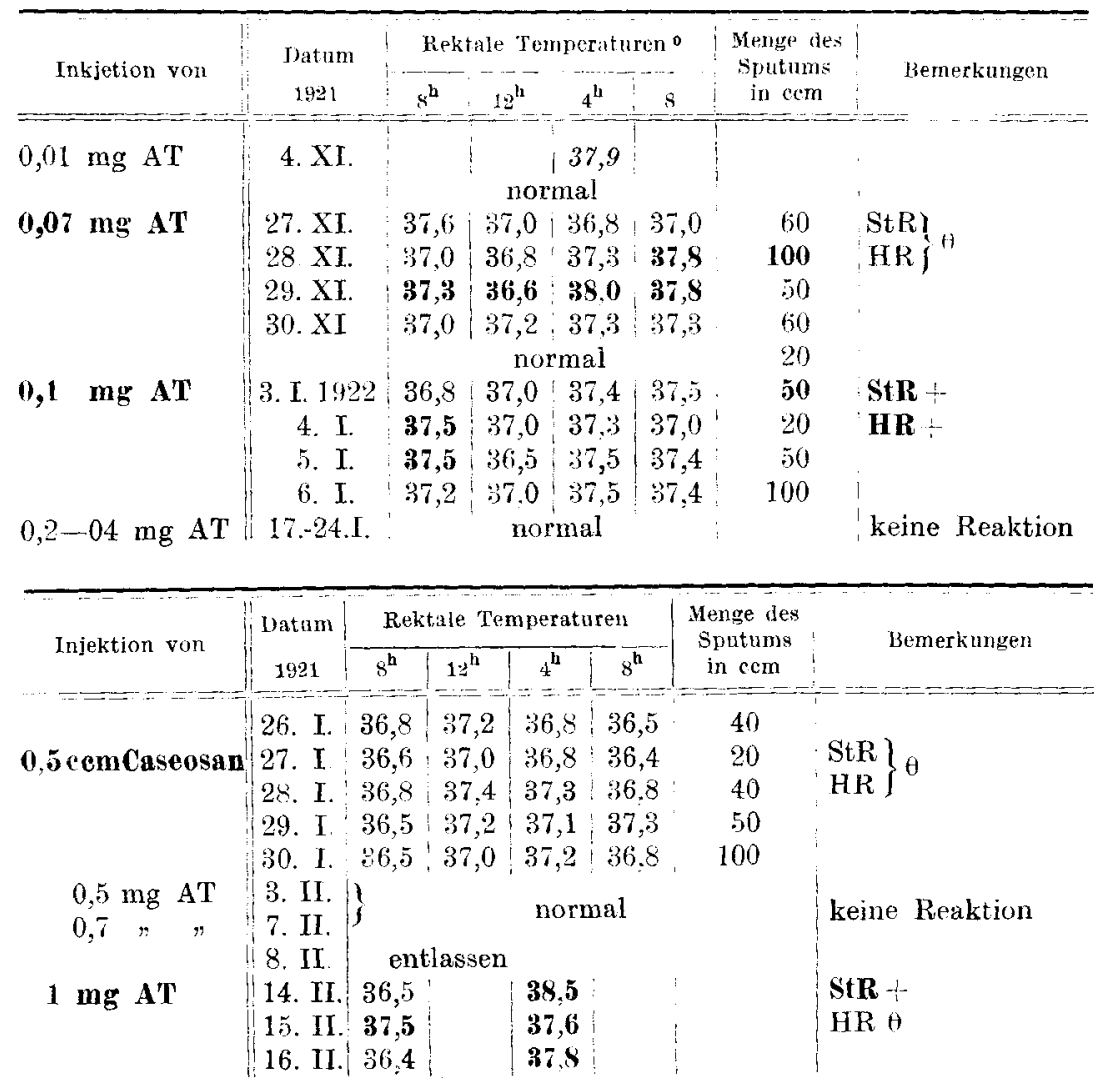

Es bleibt also die Injektion von $0,5 \mathrm{ccm}$ Caseosan ohne jede Reaktion (N-Gehalt entsprechend $100 \mathrm{mg} \mathrm{AT}$ ), während rorher 0,07 und $0,1 \mathrm{mg}$, nachher $1 \mathrm{mg}$ AT Reaktionen auslösen.

Fall Nr.6: Pe., Alfred, 23 jähriger Sattler. 23. IV. bis 25. IV.21, verlegt in Halsklinik zwecks Tracheotomie.

4. V. Wiederaufnahme, Ende März 1922 noch in stationärer Behandlung.

Diagnose: Proliferierend-indurierende Tuberkulose beider Lungen; Larynxtuberkulose.

Hereditär o. B. Taubstumm. Seit März 1921 Tuberkulose-Anamnese, starke Atemnot. - Dürftiger Ernährungszustand. Subfebril, später meist afebril. Ausgedehnte vorwiegend proliferierende Prozesse in beiden Oberlappen mit 
großer Caverne rechts oben, geringere in Unterlappen. Sputummenge zwischen 50 und $100 \mathrm{ccm}$, T.B. $\because-$ Kehlkopf: Zunächst Larynxödem infolge spezifischer infiltrativer Prozesse, keine Ulcera. Starke Stenosebeschwerden. Tracheotomie, dann durch Dauerkanüle Stillstellung des Kehlkopfs mit sehr gutem Heilungserfolg. Immunbiologisch zunächst unentschieden, schwach allergisch, später Neigung zu positiver Anergie. Lungenprozeß zeigt unter spezifischer Behandlang ganz gute Indurationstendenz.

$$
\begin{array}{clc}
\text { Gewicht bei Aufnahme } 46,7 \mathrm{~kg} . \\
" \quad \text { Mitte Marz } 53,0 "
\end{array}
$$

Therapie: Zunächst U.Tb.R., dann AT; Beginn mit $0,01 \mathrm{mg}$, durchgeführt bis $\mathrm{ro} \mathrm{mg}$.

Am 18. X. werden 60 $\mathrm{mg}$ AT, wie bisher alle Injektionen, reaktionslos vertragen. Am 1. XI. werdon 80 $\mathrm{mg}$ injiziert, dio eine derar't heftige und nachhaltige Reaktion hervorrufen, daß man von Tuberkulinschaden sprechen muB. Es folgt eine Periode hochfebriler, dann alhnählich abklingender Tomperaturen, die sich erst. Ende Norember der Norm nähern bzw. sie erreicht haben. Wenige Tage nach der Injektion setzt eine Pleuritis sicca links ein; die katarrhalischen Erscheinungen iiber beiden Lungen sind vormehrt; es entsteht eine Rekurrenslähmung, wahrscheinlich durch frisch geschwollene Drüsenpakete verursacht. Die bereits entfornte Trachealkanüle mußte wieder eingelegt werden. Das Körpergewicht nimmt um $3 \mathrm{~kg}$ ab; öfters Erbrechen. Erst im Dezember und Januar trat eine Annäherung an den alten Zustand wieder ein, wom auch in Tomporatur, Gewicht und Allgemeinbefinden sich noch eine gewisso Labilität geltend machte. Noch im Januar erfolgten Temperatursteigerungen nach mehrstündigem Aufstehen. Zur Zeit geht es dem Pationten recht gut. Es ist anzunehmon, daß nach diesem Ereignis zu Vergleichszwecken verabfolgte Caseosaninjektionen einen ungünstigen Boden vorfinden. Ich lasse jetzt die tabellarische Übersicht zum

\begin{tabular}{|c|c|c|c|c|c|c|c|}
\hline $\begin{array}{l}\text { Injektion voll } \\
\ldots\end{array}$ & $\begin{array}{c}\text { Datum } \\
4421 \\
\end{array}$ & $\begin{array}{c}\mathrm{Ke} \\
8^{\mathrm{b}}\end{array}$ & $\begin{array}{l}\text { tale } \mathrm{T} t \\
12^{\mathrm{h}}\end{array}$ & $4^{b}$ & $\begin{array}{l}\text { Iren } \\
\vdots \quad 8^{h}\end{array}$ & $\begin{array}{l}\text { Menge des } \\
\text { Sputums } \\
\text { in cem }\end{array}$ & Themerkungen \\
\hline & 31. X. & 36,5 & & 37,5 & 37,5 & 50 & \multirow{7}{*}{$\begin{array}{l}\text { Erbrechen StR } \\
\text { HR } \\
\text { Rekurrenslähm } \\
\text { Pleuritis }\end{array}$} \\
\hline \multirow{6}{*}{$80 \mathrm{mg} \mathrm{AT}$} & 1. XI. & 36,8 & 37,6 & 39,0 & 40,2 & 50 & \\
\hline & 2. XI. & 39,5 & 38,8 & 38,1 & 38,5 & 60 & \\
\hline & $3 \mathrm{XI}$. & 37,5 & 37,8 & 37,7 & 35,0 & 60 & \\
\hline & 4. XI. & 37,5 & 38,1 . & 37,8 & 38,2 & 50 & \\
\hline & ๘. XI. & 38,2 & 38,6 & 38,1 & 39,0 & 50 & \\
\hline & 6. XI. & 38,1 & 38,6 & 39,0 & $\mathbf{3 8 . 5}$ & 50 & \\
\hline
\end{tabular}
Vergleich folgen:

usw. bis 12. XI. dann wesentlich leicht- bzw. subfebril, immerhin an einzelnen Tagen, zuletzt am 26. XI. bis $39^{\circ}$. Vom 28. XI. bis Ende Dezember afebril, gelegentlich subfebril. Sputummenge um $100 \mathrm{ccm}$. 


\begin{tabular}{|c|c|c|c|c|c|c|c|}
\hline Injektion vout & $\begin{array}{c}\text { Datum } \\
1922\end{array}$ & $\begin{array}{l}\text { liek } \\
s^{\mathrm{t}} \\
\end{array}$ & $\begin{array}{l}\text { tale ter } \\
12^{\mathrm{t}} \\
\end{array}$ & nperate & $\begin{array}{r}r^{2}{ }^{0} \\
8^{h}\end{array}$ & $\begin{array}{l}\text { Henge des } \\
\text { Sputums } \\
\text { in com }\end{array}$ & Bemerkintset \\
\hline & 2. I. & 36,6 & $37, \overline{5}$ & 37,7 & 38,3 & 100 & $\begin{array}{l}\text { Aufstehen: } \\
\text { Bettruhe }\end{array}$ \\
\hline & 5. 1 . & 36,0 & 37.8 & $\hat{5}, 1$ & 37.5 & $\therefore 0$ & \\
\hline \multirow[t]{4}{*}{$1: " m$ Caseosan } & 6. I. & 36,5 & 37.9 & 37,5 & 37,5 & 100 & StR 1 \\
\hline & ‥ 1. & 36.1 & $\begin{array}{l}37,4 \\
\text { nor }\end{array}$ & $\begin{array}{l}35.2 \\
\text { nal }\end{array}$ & 37,3 & .0 & $\mathrm{HRt}$ \\
\hline & 11.1 & & & & 380 & & \\
\hline & 16. I. & 36,2 & 37,3 & 37,0 & 37,5 & 50 & \\
\hline \multirow[t]{6}{*}{ Qrem Caseosan } & 17. I. & 36,2 & 37,6 & 37,5 & 37,3 & .411 & StR " \\
\hline & 18.3 & 36,1 & 37.3 & 37,1 & 37,2 & 90 & \\
\hline & $19 . \mathrm{X}$ & 36,4 & 37.4 & 37,6 & 37,5 & 100 & :sstda. Hur HR: \\
\hline & 20.1 & 37.3 & 37,5 & 37,5 & 35,1 & (5i) & $\begin{array}{l}\text { SStd a u Hithen } \\
\text { sonire }\end{array}$ \\
\hline & & & nory & nal & & & \\
\hline & 26. & 36,9 & 37,3 & 37,1 & 37,1 & $\therefore 0$ & \\
\hline \multirow[t]{5}{*}{ 2.5eenCaseosan } & 27.1. & 36,1 & 37,3 & 34,0 & 38.1 & 50 & StR \\
\hline & $28 . \mathrm{I}$. & 37,0 & 37,5 & 37.3 & 37.3 & 70 & $H R\}^{\prime \prime}$ \\
\hline & 29. I. & 860 & 36.9 & $3 \pi, 0$ & $8 i, 3$ & 70 & \\
\hline & $30 \mathrm{I}$ & 36,5 & $\begin{array}{l}37,1 \\
\text { nom }\end{array}$ & $\begin{array}{l}37,0 \\
\text { mal }\end{array}$ & $37,2$. & & \\
\hline & is. If. & 34,3 & $3 ., 4$ & 37.3 & $: 37,1$ & 10 & 1 Stunde apt \\
\hline \multirow[t]{5}{*}{$2, \bar{b}$ cencaseosan } & 7. II. & $: 36,1$ & 37.5 & 37,4 & 34.1 & 150 & StR \\
\hline & $\therefore$ II. & 86.6 & 37.2 & 37,6 & $8 \pi, 1 i$ & 40 & HR \\
\hline & ?. 11 . & 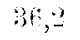 & 37,3 & 37,4 & 37,3 & 50 & \\
\hline & normal, & $\operatorname{lm} 13$ & $\begin{array}{l}\text { 11. } 38 \\
\text { Reiben }\end{array}$ & $\begin{array}{l}4^{0} \mathrm{ur} \\
\operatorname{links}\end{array}$ & d plei & ritisehes & \\
\hline & i6. II. & 396,2 & 36,8 & 37,4 & 37,5 & 160 & \\
\hline \multirow[t]{5}{*}{ 2.oc: m Caseosan } & 17.11 & 36,0 & 36,7 & 37,0 & 37.5 & 70 & StR \\
\hline & $18 . \mathrm{II}$ & 36,8 & 37,1 & 37,1 & 37,3 & 200 & $\mathrm{HR}\}^{t \prime}$ \\
\hline & 19. IT. & 36,0 & 37,2 & 37,0 & 97,4 & 110 & \\
\hline & $20.1 \mathrm{II}$ & 36,2 & $\begin{array}{l}37,2 \\
\text { nor1 }\end{array}$ & $\begin{array}{l}37,2 \\
\mathrm{nal}\end{array}$ & 37,3 & i) & \\
\hline & 24. II. & 36,0 & 37,4 & $8 \pi, 1$ & 37,4 & 60) & \\
\hline \multirow[t]{4}{*}{1 rem Aolan } & 25. II. & 56,0 & 37,8 & $3 \pi, 4$ & 37,0 & 61) & StR \\
\hline & 26. TII. & 36,0 & 37,0 & 37,3 & 37,3 & 100 & $H R\}^{\prime \prime}$ \\
\hline & 27.11. & 36,1 & 37,2 & 37,0 & 37,3 & 80 & \\
\hline & 2X. T.I. & 36,0 & 37,0 & 36.9 & 36,9 & 60 & \\
\hline \multirow[t]{3}{*}{3 cm Caseosan } & 1. III. & 36,1 & 37,2 & 37,2 & 37,6 & 90 & StR - (Oberarm, \\
\hline & 2. III. & 36,5 & 37,4 & 37,3 & 37,2 & 60) & $\mathrm{HR} \theta$ \\
\hline & 3. III. & 36,3 & 37,2 & 37.2 & $3 \pi, 0$ & 60 & \\
\hline \multirow[t]{3}{*}{$34 \mathrm{~cm}$ Caseosan } & 4. JII. & 36,2 & 37,0 & 37,2 & 37,5 & 50 & $\begin{array}{c}\text { StR }(t)(\text { ober- } \\
\text { schenke] }\end{array}$ \\
\hline & ․ III & 36,8 & 37,6 & $3 \pi, 4$ & 37,4 & 100 & $\mathrm{HR} \theta$ \\
\hline & 6. III. & 37,2 & $\begin{array}{r}37,3 \\
\text { norl }\end{array}$ & $\begin{array}{l}37,3 \\
\text { nal }\end{array}$ & $37,5 !$ & 100 & i $\mathrm{g}<$ früher. \\
\hline
\end{tabular}

Die Reaktionen, die auf steigendo Caseosanmengen erfolgen. sind nicht hochgradig und werden trotz Dosensteigerung allmählish geringer. Ihre N-Mengen entsprechen dem mehr als Doppelten bis 
6fachen der AT-Dosis ( $80 \mathrm{mg}$ AT entspricht $0,376 \mathrm{ccm}$ (aseosan). Wir scheuten uns in diesem Fall, nochmals mit AI die Allergieverhälnisse zu überprüfon. Nach don Erfahrungen der letzten Injektion würde es ein Wagnis bedeutct haben, dem Caseosan-N entsterhend etwa 2015-6m) mg AT zu injizioren. Immerhin zeigt der Fall, daB man mit dieson EiweiBdosen in empfudlichen Herden Reaktionen auslösen kann.

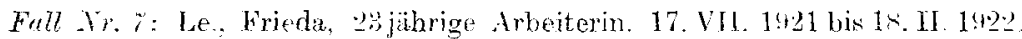

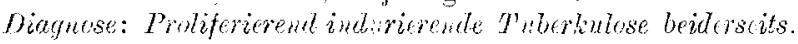

Hereditär o. B. 1920 (rippo, anschliebend Tuberkulose-Anamnese. 1) irftiger Frmährungszustand. Tenperatur subfebril-fabril mit normalen Interralen. Ziemlich ausgedelinte produltiwe und eirrhotische Prozesse. Kleine (avernen: Pleurasehwarte links. Sputummenge durelschnitilich un $50 \mathrm{~cm}$. T.B. . Inmunbiologisch: allergikeh, statk tuberkulinemptindlich. Geringe: Besserung, klingende Rasselgeräusche nach AbschluB der Bohandlung vershwunden

$$
\begin{gathered}
\text { Gewioht hei Autmahne }+1, \overrightarrow{\mathrm{i}} \mathrm{k}, \\
. .
\end{gathered}
$$

Thrapie: Atr, Beginn zunächst mit 0.1 , dan 0.01 me. "ber 2 mg himans nicht durchtïhrbar.

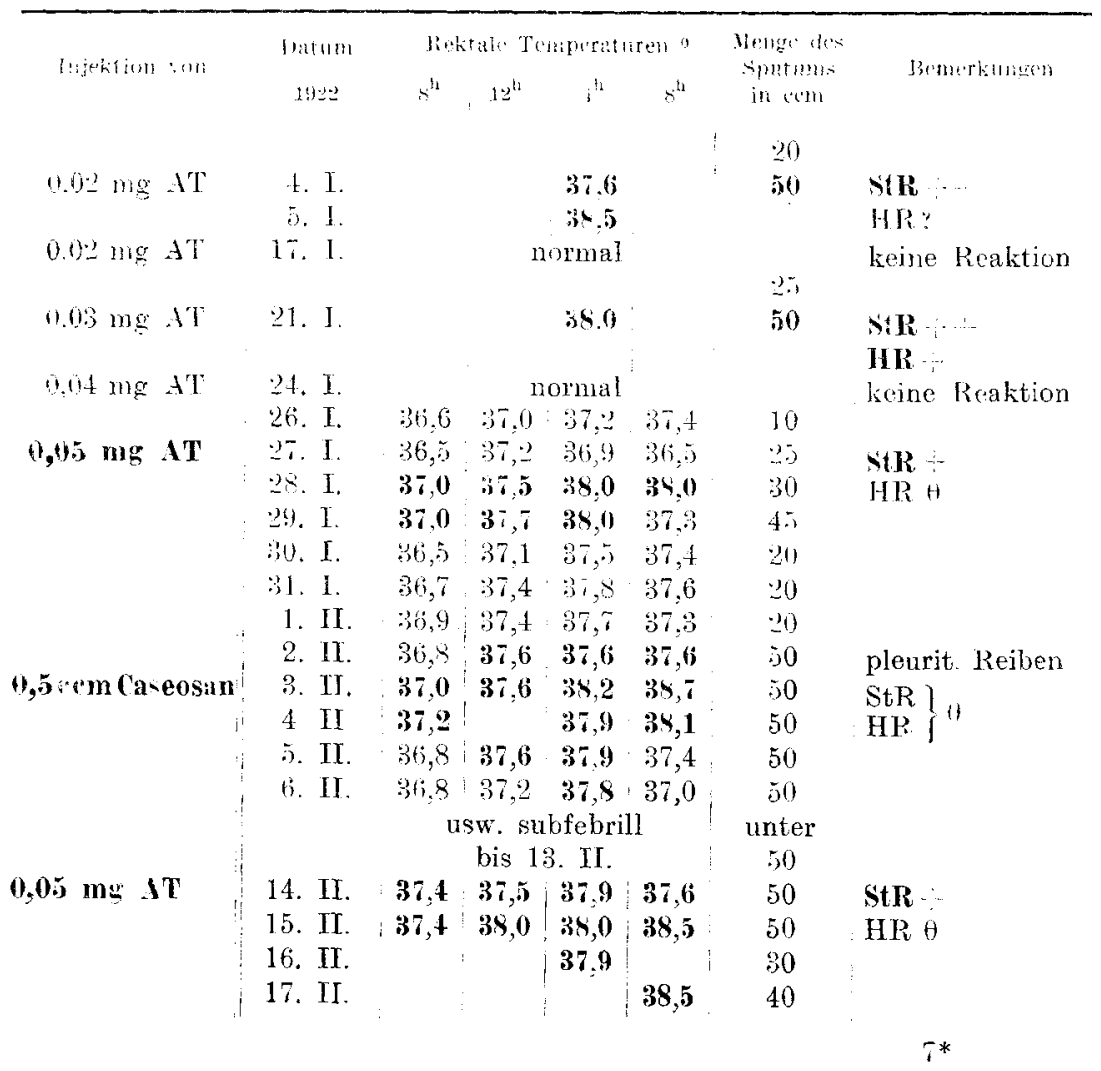


Der Fall ist schwel zu beurteilen. An und für sich unterbrechen schon ständig Temperatursteigerungen die normalen Perioden; es bestehen sicher neben älteren ganz frische reaktive Prozesse. Dazu gesellt sich eine starke neurasthenische Komponente: die Patientin war oiner Injektionstherapie sehr abgeneigt.

Der Fall ist für unsere Fragentellung kaum verwertbar. Man vermißt die Vergleichsprüfung mit kleineren Caseosandosen. Die Dosis von $0,5 \mathrm{~cm}$ Caseosan ( $\mathrm{N}$-Dosis entspricht $100 \mathrm{mg}$ AT) war entschieden für einen Vergleich riel zu hoch gewählt (200)fach) und außerdem zu einer ungeeigneten Zeit, nämlich einen Tag nach dem Einsetzen einer frischen Pleuritis, einverleibt. Sie stellt einen Reiz für den empfindlichen Organismus dal, der cine ganz gleiche Reaktion auslöst wie die umrahmenden AT-Dosen von 0,05 mg. Immerhin darf zugegeben werden, daß - nach Kenntnis der oben gezeigten Tuberkulinempfindlichkeit - die entsprechende Dosis ron $100 \mathrm{mg}$ AT einen kaum gut zu machenden Tuberkulinschaden heraufbeschwören müßste.

Auch der folgende Fall entspricht in seiner Versuchsinordnung nicht der eigentlichen Fragestellung der Arbeit. Da er jedoch gut zu den Fällen der 3. Gruppe überleitet. sei er hier angeführt:

Fall Nr. 8: Lu., Wilhelm, 21 jähriger Schlosser, 21. I. bis 1. III. 1922.

Diagnose: Proliferierend-indurierende Tuberkulose beidler Oberlappen.

Hereditär o. B. Seit Grippe Juni 1921 Tuberkulose-suspekte Anamnese. Mäßig ernährt, afebril. Klinisch und röntgenologisch vorwiegend cirrhotische, nicht sehr ausgedehnte Prozesse besonders am Hilus beiderseits. Sputummenge zwischen 20 und $50 \mathrm{com}$, T.B. (nach Antiformin) spärlich + . Nach Behandlung Katarrh fast verschwunden. Immunbiologisch: Allergisch mit Neigung zu poiitiver Anergie.

Gewicht bei Aufuabme $53,6 \mathrm{~kg}$,

$$
" \quad \text { "Entlassung 56,. " }
$$

Therapie: AT, Beginn mit $0,001 \mathrm{mg}$; ambulant bisher bis $0,04 \mathrm{mg}$.

\begin{tabular}{|c|c|c|c|c|c|c|c|}
\hline \multirow{4}{*}{$\begin{array}{l}\text { Injektion von } \\
=-=-== \\
0.001 \mathrm{mg} \mathrm{AT}\end{array}$} & Datum & \multicolumn{4}{|c|}{ Rektale Temperaturen" } & Menge des & \multirow{2}{*}{ Bemerkungmn } \\
\hline & 1922 & $8^{\mathrm{h}}$ & $12^{\mathrm{h}}$ & $4^{b}$ & $\mathrm{x}^{\mathrm{b}}$ & in $\mathrm{cem}$ & \\
\hline & 7. II. & & & & & & \\
\hline & 9. II. & & & $3 \overline{7}, \overline{7}$ & & 20 & \\
\hline \multirow[t]{3}{*}{$0,002 \mathrm{mg} \mathrm{AT}$} & 10. II. & & & & & & \\
\hline & 12. II. & & & 37,8 & & 20 & \\
\hline & 13. II. & 36,0 & 37,3 & 37,3 & $3 \pi, 2$ & 10 & \\
\hline \multirow{3}{*}{$0,004 \mathrm{mg}$ AT } & 14. II. & 36,2 & 37,3 & 37,4 & 37,2 & 10 & StR $\div$ \\
\hline & 15. II. & 36,3 & 37,5 & 37,8 & 38,2 & 10 & HR \\
\hline & 16. II. & 36,8 & 36,8 & 36,8 & 36,7 & 10 & \\
\hline \multirow{4}{*}{1 cem Caseosan } & 17. II. & 36.3 & 37.9 & 38,5 & 39,5 & 15 & Alte AT-StR \\
\hline & 18. II. & 37,4 & 37,1 & 37,3 & 37,3 & fehlt & $\mathrm{HR} H(\mathrm{Rg} \mathbf{r}=\mathrm{I} H)$ \\
\hline & 19. II. & 37,7 & 38,3 & 37.8 & 37,5 & 40 & \\
\hline & 20. II. & 37.1 & $\begin{array}{r}38,0 \\
\text { no }\end{array}$ & $\begin{array}{l}37,3 \\
\text { mal }\end{array}$ & 36.9 & 30 & \\
\hline
\end{tabular}


Blutbild

a) vor: der Caseosaninjektion:

Leukocyten 8900 , davon

Polynucl. neutroph. $70^{0}$,

Lymphocyten $22^{\circ}: 0$,

Mononucl. $\quad 3 \%$,

Übergangsf. $\quad 3 \%$,

Eosinoph. $\quad 2^{0 !}$.

b) $6^{\text {h }}$ nach der Injektion (Höhe der Reaktion):

Leukocyten 9400, davon

Polynucl. neutroph. $84^{0} 0^{\circ}$,

Lymphocyten $\quad 9 \%$,

Mononucl. $\quad 5^{0}$,

Übergangsf. $\quad 0 \%$,

Eosinoph. $\quad 20 / 0$.

An dieser Stelle sei bemerkt, daß die in mehreren Fällen vor nnd nach AT- und Caseosaninjektionen untersuchten Blutbilder im allgemeinen keine deutlich verwertbaren Verschiebungen zeigten. Man kann rielleicht sagen, da\} reaktionslose Caseosandosen auch keine Blutbildsveränderungen machen, während bei Auftreten einer fieberhaften Reaktion eine leichte polynucleäre Leukocytose beobachtet werden konnte.

AT machte bisweilen deutliche Lymphocytose, auch bei Fehlen sonstiger Reaktionen (siehe z. B. Fall Nr. 12, Jh.; ebenso Fall Nr. 1. La., bei $10 \mathrm{mg}$ um $10 \%$ von 22 auf $32 \%$.

Naegeli hält die bei Tuberkulosen mit Heilungstendenz durchweg beobachtete Lymphocytose für den Ausdruck der Uberwindung des toxisch-infektiösen Moments, nicht für ein Zeichen der Chronizität. Seit Bergels experimenteller Begründung der Lymphocytose sind wir geneigt, mit ihm anzunehmen, daß die lymphocytären Elemente des Bluts elektiv chemotaktisch auf Krankheitserreger lipoiden Charakters reagieren.

Bergel hält sinngemäß die wahllose künstliche Erzeugung einer polymorphkernigen Leukocytose durch Proteinkörpertherapie bei verschiedensten Krankheiten für fehlerhaft.

Wenn Weicksel, wie oben erwähnt, dem Caseosan die Eigensehaft, öfters zu stark reizend zu wirken, zuschrieb, so geschah das anch mit besonderer Berücksichtigung der Lymphocytenkurve. Wo infolge Uberdosierung eine Verschlechterung als Folge der Injektionen eintrat (beim AT wegen seiner bekannten Dosierung seltener als beim Caseosan), fand er besonders bei schwereren Fällen Iymphocyton sturz als ungünstiges Zeichen. 
Schulz fand Lymphocytose nach Tuberkulininjektionen als kon. stanten Befund. Bergel betont, daß therapeutisch gïnstig wirkende Prozeduren wie neben dem Tuberkulin die Höhensonne, der Pneumothorax, Fettnahrung, Jod, Lebertran, Stauung, Höhenluft auch Lymphocytosen erzeugen.

Diese Annahmen und Ergebnisse, die sich mit unsern Befunden decken, scheinen danach sämtlich für eine spezifische Wirkung des Tuberkulins auf das Bluttild zu sprechen, die dem Caseosan algeht.

In weiteren Verlauf der Therapie wurden am 22. II. 0,005 mg AT und $0,007 \mathrm{mg}$ am 25. II. reaktionslos vertragen. Am 29. II. wurde Pat. aus der stationären Behandlung entlassen und kam weiter ambulant:

\begin{tabular}{|c|c|c|c|}
\hline $\begin{array}{l}0,01 \mathrm{mg} \\
0.02\end{array}$ & 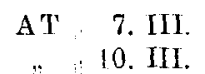 & $\begin{array}{l}\text { normal } \\
\text { dgl. }\end{array}$ & keine Reaktion \\
\hline 0,04 & 14. III. & $3 \mathcal{S}^{\circ}$ & $\begin{array}{l}\text { St } R: \\
\text { HR: vermehrter Husten }\end{array}$ \\
\hline
\end{tabular}

In diesem Fall macht die Injektion von $1 \mathrm{ccm}$ Caseosan eine beträchtliche Allgemein- und Stichreaktion am 17. II., beides stärker als die reaktiven AT-Dosen von 0,004 und später 0,04 $\mathrm{mg}$ (N-Gehalt des Caseosans $50000-5000$ fach), dagegen sicher keine Herdreaktion (Kopfschmerzen und Allgemeinerscheinungen, aber kein vermehrter Husten, keine Rasselgeräusche) wie das AT vorher und vielleicht auch am 14. III. Die Pause zwischen den beiden Injektionen $(0,004 \mathrm{mg}$ A'T und $1 \mathrm{ccm}$ Caseosan) - ein fieberfreier Tag - - ist zu kurz.

Fall Nr. 9: Gi. Max, 42 jähriger Arbeiter. Seit Oktober 1920 mehrfach in stationärer Behandlung der Klinik, zuletzt vom 12. I. bis 16. III. 22.

Diagnose: Proliferierend-indurierende Lungentbc. vorw. $r$. Status nach paravertebraler Rippenresektion $r$. (zweizeitig Februar 1921).

Familienanamnese o. B. Seit 1918 lungenkrank. - MäBiger Ernährungszustand. Oktober 1920 zunächst hochfebri], dann mit zunehmender Indurationstendenz des vorwiegend rechtsseitigen Prozesses Entfieberung und (iewichtszunahme. Pleuraschwarte r., Schrumpfung der r. Thoraxseite. Nach paravertebraler Rippenresektion zunächst wieder febril, bis Juni 1.221 dann afebril. Sputummenge um $50 \mathrm{ccm}$, T. B. -t, später ti. Januar 1922 nach Verschlechterung (Grippe) wicder fcbril aufgenommen. duch links produktive Herde. Hümoptoe. Von Anfang Februar an Besserung, afebril. Sputummenge um $100 \mathrm{ccm}$, T. B. +. Neigung zu positiver Anergie. - Tertiäre Lues.

Therapie: AT von Juli bis Sept. 1921 bis $40 \mathrm{mg}$. Anfang Februar 1922 Beginn mit $0,001 \mathrm{mg}$ (s. folgende Tabelle).

Leichte Allgemein- und Stichreaktion, vermehrte Sputummenge nach $1 \mathrm{com}$ Caseosan; keine AT-Reaktion. 


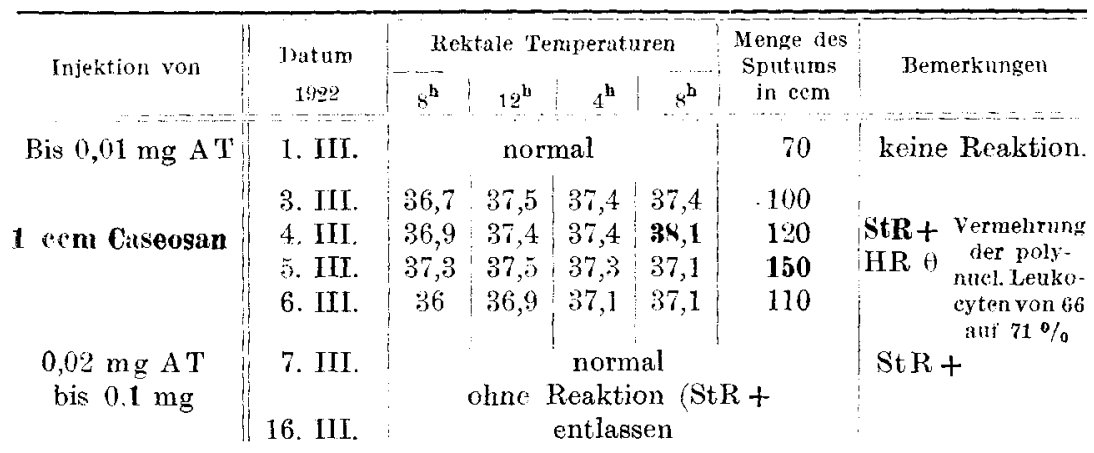

Fall Nr. 10: B1., Ferdinand, 62jähriger Arbeiter. 23. I, 22- noch in stationärer Behandlung.

Diagnose: Proliferierend-induriesende Tuberkulose beider Oberlappen.

Hereditär o. B. $191 \varangle$ Lungenentzündung, 1921 leichte Grippe. Anfang Januar 1922 Hämoptoe. -- Mäßiger Ernährungszustand. Im Beginn leicht febril, dann afebril. Wenig ausgedehnte produktive Herde über beide Spitzen und in der Hilusgegend, mit guter Indurationsneigung. Sputummenge zwischen 50 and $100 \mathrm{ccm}$, T. B. +. Immunbiologisch: Neigung zu positiver Anergie.

$$
\begin{gathered}
\text { Gewicht bei Aufnahme } 66,0 \mathrm{~kg} \text {, } \\
\text { Ende März 70,0 ", }
\end{gathered}
$$

Therapie: A T, Beginn mit 0,001 $\mathrm{mg}$, bisher bis $0,7 \mathrm{mg}$.

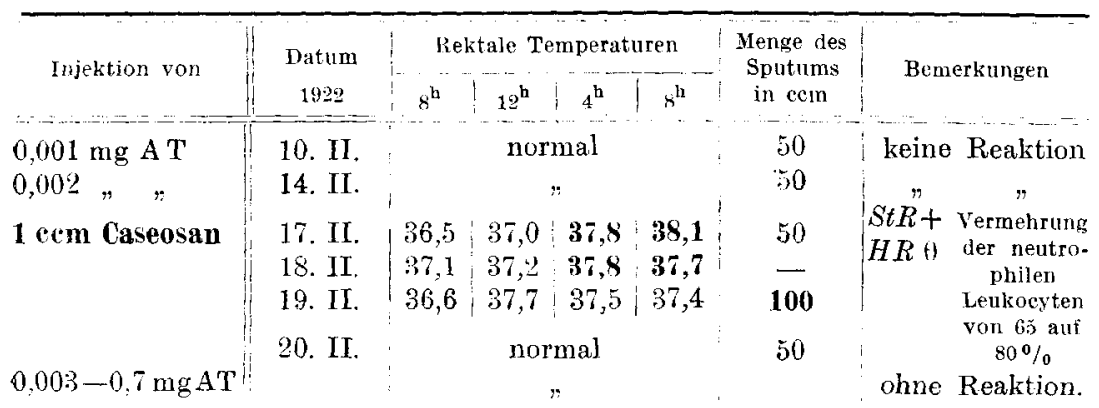

Wie der vorhergehende Fall.

Fall Nr. 11: We., Adolf, 24 jähriger Arbeiter 13. II. 22 - noch in stationärer Behandlung.

Diagnose: Proliferierend-indurierende Tbc., vorwtegend der $r$. Lunge.

Hereditär o. B. Seit Herbst 1921 lungenkrank. - Mäßig ernährt; kurze Zeit leicht febril, dann afebril. Im Bereich der rechten Lunge (Ober- und Mittellappen mehr als Unterlappen) produktive und indurierende Herde in ziemlicher Ausdehnung, 1. bedeutend geringer. Sputummenge zwischen 20 und $50 \mathrm{ccm}$. T. B. . Immunbiologiseh: allergisch, Nejgung za positiver Anergie.

$$
\begin{gathered}
\text { Gewicht bei Aufnahme: } 55,3 \mathrm{~kg}, \\
\text { Ende März } 57,5 \mathrm{~m},
\end{gathered}
$$

Therapie: A T, Beginn mit 0,001 $\mathrm{mg}$, bisher bis $0,8 \mathrm{mg}$. 


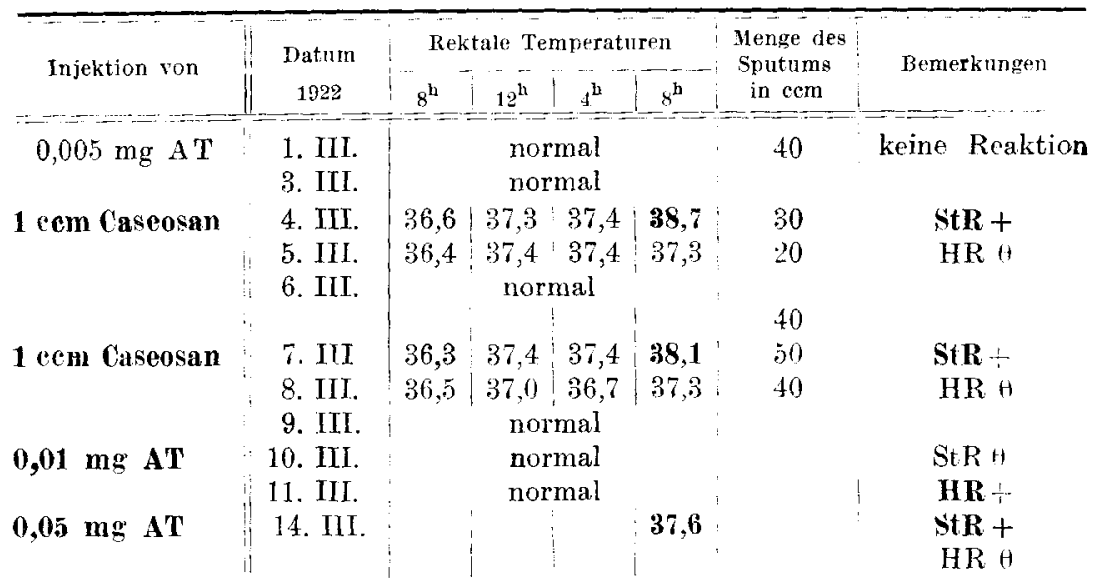

Auf $1 \mathrm{ccm}$ Caseosan beide Male leichte Allgemein- und Stich-, beine Herdreaktion. $0,01 \mathrm{mg} A \mathrm{~T}\left(\mathrm{~N}={ }^{1 / 20000}\right.$ des Caseosans) macht sichere Herdreaktion.

Fall Nr. 12: Th., Gustav, 36 jähriger Dreher. 20. I. bis 15. III. 22.

Diagnose: Vorw. rechtsseitige proliferierend-indurierende Lungentbc.

Vater an Lungenentzündung gestorben. Seit Sommer 1920 lungenkrank. - Mittelernährt, afebril. Nicht sehr progresse produktive Herde im Bereich des r. Oberlappens mit guter Indurationstendenz. Sputummenge zwischen 50 und $100 \mathrm{cem}$, T. B. +. Immunbiologisch: allorgisch mit Neigung zu positiver Anergie.

Gewicht bei Aufnahme $54,7 \mathrm{~kg}$, bei Entlassung 56,3 ,

\begin{tabular}{|c|c|c|c|c|}
\hline Injektion von & Batum $\begin{array}{c}\text { Bam } \\
1922\end{array}$ & $\begin{array}{l}\text { Rektale Temperaturen } \\
8^{\mathrm{h}}-12^{\mathrm{h}}-4^{\mathrm{h}}\end{array}$ & $\begin{array}{l}\text { Menge des } \\
\text { Sputums } \\
\text { in cem }\end{array}$ & Bemerkungen \\
\hline \multirow{3}{*}{ Bis $0,1 \mathrm{mg} \mathrm{AT}$} & 1. III. & normal & 40 & keine Reaktion \\
\hline & 2. III. & $n$ & 70 & \\
\hline & 3. III. & $n$ & 50 & \\
\hline \multirow[t]{3}{*}{$1 \mathrm{ccm}$ Caseosan } & II 4 III. & $\eta$ & 100 & StR + \\
\hline & 5. III. & $"$ & 100 & $\mathrm{HRH}$ \\
\hline & 6. III. & $"$ & 70 & \\
\hline \multirow{2}{*}{3 rcm Caseosan } & 7. III. & $"$ & 100 & StR + \\
\hline & $\begin{array}{l}\text { iI } \\
\text { if. III. } \\
\text { 9. III. }\end{array}$ & $"$ & $\begin{array}{l}100 \\
100\end{array}$ & $\mathrm{HR} \theta$ \\
\hline \multirow[t]{3}{*}{$0,2 \mathrm{mg} \mathrm{AT}$} & 10 . III. & $n$ & 60 & StR + \\
\hline & 11 . III. & $n$ & 50 & $\mathrm{HRH}$ \\
\hline & 1. 14. III. & Aus disziplinar & Gründen & entlasseu. \\
\hline
\end{tabular}

1. III. Blutbild:

a) vor Injektion von $0,1 \mathrm{mg} \mathrm{AT}$ 5200 Leukozyten, davon polynucl. neutroph. $76 \%$ Iymphosten $\quad \mathbf{2 2} \%$ Eosinoph. Mononucl. Ùbergangsf. b) $6^{\mathbf{b}}$ nachher 5900 $56 \%$ $39 \%$ $1 \%$ $3 \%$ $1 \%$ 
4. III. Blutbild:

a) vor Injektion von $1 \mathrm{ccm}$ Cascosan 6200 Leukozyten, davon

b) $6^{\text {th }}$ nachher polynucl. neutroph. $66^{\circ}{ }_{0}^{\circ}$

7000

Lymphocyten $\quad 28^{\circ}{ }_{0}$

$68 \%$

Eosinoph.

Mononuel.

Utbergangsf.

$$
5 \%
$$

$26 \%$

-

$4 \%$

$2 i_{0}$

\section{IIT. Blutbild:}

a) vor Injektion von $2 \mathrm{ccm}$ Caseosan

5800 Leukozyten, davon

polynucl. neutr. $\quad 650$ :

Lymphocyten $290^{\circ}$

Eosinoph.

Mononucl.

Ubergangsf.

b) nachher

5200

670

270

$-$

$\therefore 0$.

1. "॥"

$\mathrm{Zu}$ den Blutbildsveränderungen s. Bemerkungen zu Fall Nr.s. Im übrigen ein Fall, der sich Injektionen von 1 und 2 com Caseosan gegenüber refraktär verhält, dessen Reaktion auf $\mathrm{AT}$ leider nicht durchgeprüft werden konnte, da seine Entlassung vorher elfolgen inußte.

Fall Nr. 13: B1., Emil, 21 jähriger Schweizer. 27. X. 21 bis 1. I1. 2.2.

Diagnose: Caceruöse Lungenphthise (Mischform). Larynx-Tbe.

Hereditär o. B. Seit 1918 lungenkrank. - Leidlich ernährt; febril wis $38,5^{\circ}$ ). Ausgedehnte Prozesse in beiden Lungen, bes. r. produktiv-cirrhotischer Art mit grober Caverne r. o.; 1. frischere Prozesse, teils wohl auch acinoskäsiger und sublobulär-käsiger Natur. L. kleineres Cavum. T'lceröse Larynxtbe. Sputummenge um $100 \mathrm{cem}$, T. B. +. Immunbiologisch: allergisch.

$$
\begin{array}{r}
\text { Gewicht bei Aufnahme: } 62,4 \mathrm{~kg} \text {, } \\
\text { bei Entlassung: } 62,3 \mathrm{~kg} \text {. }
\end{array}
$$

Therapie: Symptomatisch, kleine Caseosandosen $(0,1-0,5 \mathrm{~cm})$ subeut, Krysolgan $0,05-0,1$ iv als Larynxtherapie.

Es würde zu weit führen, die Fieberkurve im ganzen tabellarisch wiederzugeben. Deutliche Einflüsse des Caseosans auf die Temperaturbewegung sind nicht zu bemerken. Pat. erhielt am 15. XI. $0.1 \mathrm{ccm}$ Caseosan, höchste Abendtemp. $38,6^{\circ}$ gegen $38,2^{\circ}$ am Vortage; am 29. XI. $0,2 \mathrm{ccm}\left[28.38,1^{\circ} ; 29,38,1^{\circ}\right] ;$ am 3. XII. 0,3 $[38,2-38,2]$; am 6. XII. 0,4 [38,0-38,0]; am 12. XII. $0,5 \quad[37,7$ -38,2]. Weitere Caseosanmengen erhielt er nicht. Die Auswurfmenge ist $1-2$ Tage nach $0,3 \mathrm{ccm}$ um $20 \mathrm{ccm}$ vermehrt, sonst unbeeinflußt. Leichte Stichreaktionen waren jedesmal vorhanden. Eine Herdreaktion konnte nicht beobachtet werden; es scheint, als ginge der Katarrh allmählich während der Zeit von Mitte November bis Mitte Dezember zurück, wie auch die Temperaturkurve oher cine leichte Neigung zur Norm zeigt. Das Gewicht nahm auf $64 \mathrm{~kg}$ zu. Es folgten dann die Krysolganinjektionen. Von ihnen hatte ich 
(wie auch in anderen Fällen) den deutlichen Eindruck eines ungünstigen Einflusses auf empfindliche Lungenherde. Trotzdem es nicht zum eigentlichen 'Thema der Arbeit gehört, sei es hier er'wähnt, weil es dem Caseosan gegenüber eine gewisse Vergleichsmöglichkeit gewährt.

13. XII.

14. XII. $0.05 \mathrm{Krysolgan}$

15. XII.

16. XII.

17. XII.

20. XII.

21. XII

32. XII.

23. XII.

24. XII.

$\begin{array}{cc}\text { Höchste Tagestemperatur: } & \text { sput um } \\ 37,9^{\circ} & 100 \\ 38,4^{\circ} & 100 \\ 38,7^{\circ} & 100 \\ 38,7^{\circ} & 120 \\ 38,3^{\circ} & 150 \\ 37,8^{\circ} & 100 \\ 38,6^{\circ} & 100 \\ 38,7^{\circ} & 100 \\ 37,7^{\circ} & 100 \\ 37,7^{\circ} & 150\end{array}$

und in ähnlicher Weise fort bis zum Absetzen der Therapie. Der Husten war stärker, das Allgemeinbefinden schlechter, Gewicht fiel auf $62,1^{\circ}$; Herdreaktionen nicht sicher.

Dagegen kann man dem Caseosan keinen merklich ungünstigen Einfluß nachweisen. In diesen Mengen scheint es selbst auf empfindliche Herde nicht stark reaktiv zu wirken.

lch möchte nicht verfehlen, den Assistenten der Klinik, die nach mir Stationsärzte der Tuberkulosestation waren, Herrn Dr. Schmid von Neidhardt und Herrn Dr. Graf, welche die vergleichenden Injektionen in meinem Sinne fortführten, für ihre Mitarbeit meinen Dank auszusprechen.

\section{Kritik der Versuchsresultate.}

Die Ergebnisse beider Versuchsreihen sprechen eindeutig dafür, daß ein aus der Errechnung gleichen N-Gehalts im Tuberkulin und im vergleichsweise injizierten Proteinkörper gewonnener Maßstab eine ausgesprochene Uberlegenheit der reaktiven Wirkung des Tuberkulins erweist.

Im Kochschen Hauptversuch gehen die mit $0,3-0,5 \mathrm{ccm}$ AT oder AF-Stammlösung ip gespritzten Tiere prompt zugrunde, während entsprechende Mengen von Eiweißkörpern ohne Effekt auf Leben und Gesundheitszustand der Tiere bleiben.

Die vergleichenden Injektionen am tuberkulösen Menschen lassen sich eigentlich nur im Zusammenhang des Reaktionsablauts, d. $h$. mit Berücksichtigung des sich ständig verschiebenden immunbiologischen Zustandsbildes beurteilen und sich schwer in einer Synopsis nach $f$ und - zusammenbringen. Doch sei der Versuch gemacht. Es sind dabei von positiven AT-Injektionen nur die unmittelbar der Caseosaninjektion vorausgehenden oder die nachzeitigen berücksichtigt. 
Die Spezifität der Tuberkulinreaktion.

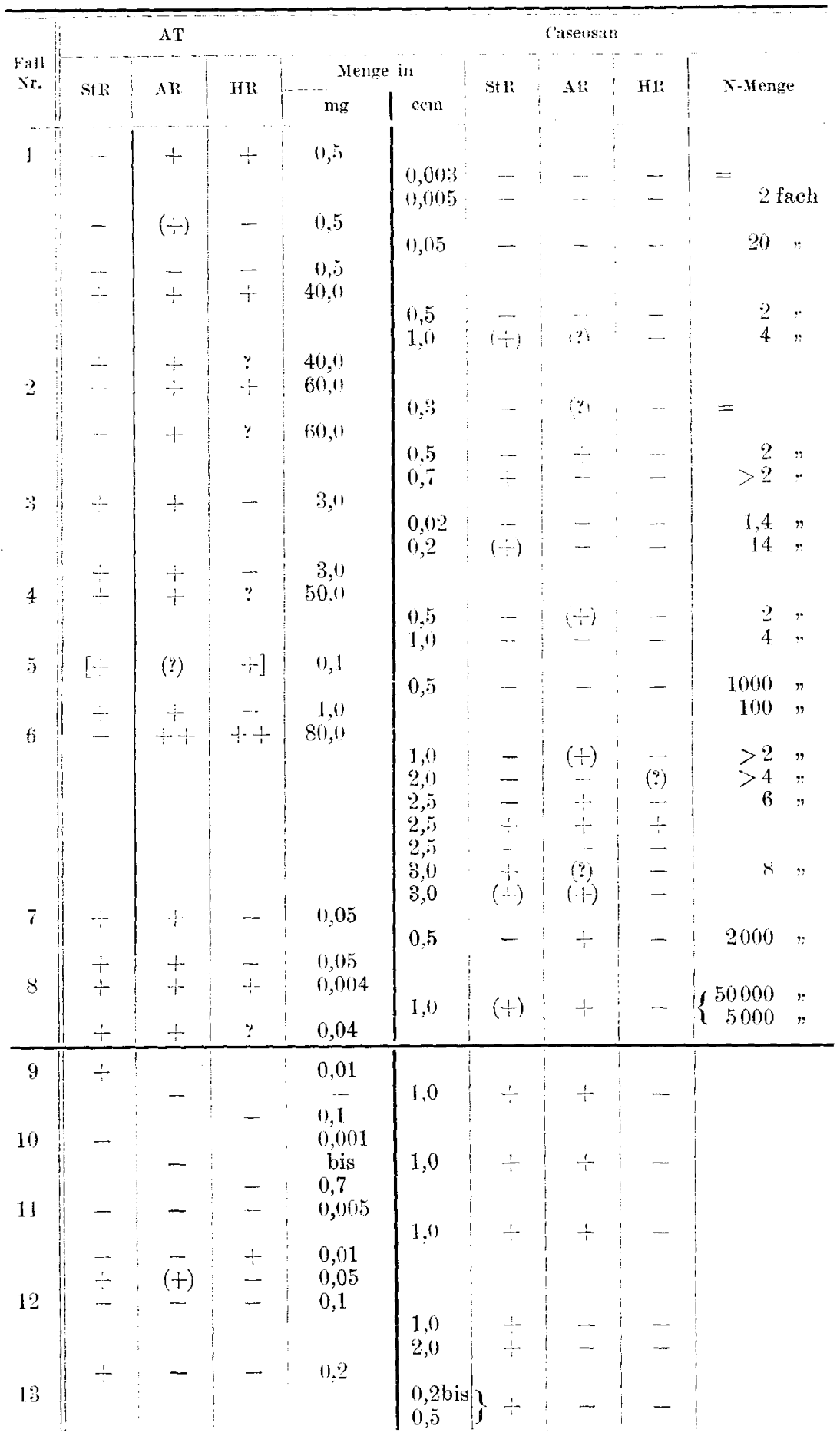


Es ergibt sich aus dieser Gegenüberstellung, daß das Caseosan bei gleichem $\mathrm{N}$-Gehalt nie, aber auch bei doppeltem und vielfachem bei weitem nicht immer Reaktionen auslost, wo durch AT solche hervorgerufen wurden. In Mengen von $0,5-3,0 \mathrm{ccm}$ kann es Reaktionen auslösen, die denen des AT gleichen, unter denen aber nur eine sicher beobachtete Herdreaktion sich findet. Dieser Unterschied erscheint mir sehr wesentlich. Daß Tuberkulöse an und für sich leicht fieberhaft auf beliebige Reize reagieren, bietet keine Besonderheiten. Sie scheinen sich hierin übrigens kaum von andern Kranken mit entzündlichen Affektionen zu unterscheiden. Bier betont die erhöhte Reizbarkeit, die Entzündungsherde vor dem gesunden Gewebe auszeichnen, so daß Proteinkörper auf Kranke ganz anders als auf Gesunde wirken. Für die individuell sehr verschiedenen Reizschwellen lassen sich wohl kaum Standardzahlen aufstellen. Alle Autoren betonen die Schwierigkeit der Dosierung von Proteinkörpern (Zimmer. Lindig u. a.). Arweiler fand, daß $0,05 \mathrm{ecm}$ Casein bei gynäkologisch Kranken Fieber hervorrufen kann; das würde also einer Menge von $1 \mathrm{ccm}$ Caseosan-Heyden ( 5 proz. Caseinlösung) entsprechen. Dosen unter $0,5 \mathrm{ccm}$ scheinen bei Tuberkuläsen nicht reaktiv zu sein.

Es scheint mir nach dem Gesagten nicht möglich, dem Caseosan eine ähnlich elektive Wirkung wie dem Tuberkulin zuzuschreiben. Vielleicht ist die Tuberkulinreaktion zum Teil eine Proteinkörperreaktion; darin mag auch die äußere Ähnlichkeit beider begründet sein. Das Tuberkulin ist dem Eiweißkörper jedenfalls in den beiden wichtigsten Punkten überlegen: in dem gesetzmäßigen Uberschreiten der Reizschwelle, unter. Umständen auch mit kleinsten Dosen, unabhängig rom $N^{\dagger}$ Gehalt, und in der stärkeren Affinität zum spezifischen Herd.

Dabei ist heute immer noch nicht in verneinendem Sinne über die Frage entschieden, ob mit der bloßen Reizwirkung des Tuberkulins, die ja schließlich auch die Proteinkörper, wenn auch in gröberem Maße, mit ihm gemein hätten, seine Wirkungsweise ersehöpfend erk]ärt ist.

Therapeutisch wird man die elektive, fein abstufbare Wirksamkeit des Tuberkulins nicht missen wollen, falls man eben nicht gerade von der geringeren Affinität der Proteinkörper zum spezifischen Herd in frisch-proliferierenden oder exsudativen Fällen Gebrauch machen will. Dabei scheint mir immer noch fraglich, ob man mit den verwendbaren kleinen Dosen überhaupt Effekte erzielt oder unter der Reizschwelle bleibt. Es sei ferner noch oinmal an die Beoinfussung des Blutbilds und die prognostische Bedeutung seiner symptomatischen. Veränderungen erinnert, die auch nicht für die Proteinkörpertherapie bei Tuberkulose sprechen. Ich glaube kaum, daß man in derartigen 
Fällen mit Proteinkörpern Besseres erzielen kann als mit intracutaner Appikation kleinster Tuberkulindosen, wie z. B. bei der MTbR-Themapie, die schon in der Haut spezifische Umstimmungen hervorrufen.

\section{Zusammenfassung.}

1. AT- und Caseosaninjektionen von gleichem N-Gehalt haben auf tuberkulöse Individuen, Meersehwoinchon wie Menschen, eine durchaus verschiedene Wirkung: erst Caseosandoson von weit höherem X-Gehalt als entsprechende AT-Dosen machen ähnliche Reaktionen.

2. In den verglichenen Mengen (bis zu 3 cen Caseosan) macht das Casoosan wohl flüchtige Stich-, mäBige Allgemein-. aber fast niemals Herdreaktionen.

3. Mengen untor 0.5 cem Caseosan scheinen unwirksam.

4. Es geht die Spezifität des Tuberkulins aus seiner außerordentlich fein abgestuften Wirksamkeit auf empfindliche tuberkulösc Horde hervor, die sich unterUmständen auch beikleinsten Mengen in hohem Maße äußert.

5. In der Therapie der Tuberkulose verdient daher das Tuberkulin vor den schwer dosierbaren Proteinkörpern den Vorzug.

\section{Literaturverzeichnis.}

Arueiler, Therap. Halbmonatshefte 1920, H. 17. - Bergel, Ergebn. d inn. Ned. u. Kinderheilk. 20. 1921. - Bessau, Jahrb. f. Kinderheilk. 1915. Nr. 5 u. 6; Münch. ned. Wochenschr. 1915, Nr. 11). - Bier. Münch. med. Wochenschr. 19:1. Nr.6 u. 14. - Buchner, Münch. med. Wochenschr. 1×91. Nr. 49. - Doutrelepont, Klin. Jahrbuch. Erg.-Bd. 1\$91, S. 3*2; Dtsch. med. Wochenschr. 190x. S. 263. -- Engel und Bauter, Beitr. z. hlin. d. Tuberkul. 13, Nr. 3. - v. Hayeh: Wien. klin. Wochenschr. 1920, Nr. 35 u. 36; Tuberkuloseproblem. Springer. 1921, ¿. 99 fi. Kaznelson, Therap. Halbmonatshefte 1917, S. 437; Weichardts Ergebn. IV. .. Klemperer, Berl. klin. Wochenschr. 1920. Nr. 45 u. 46. -.- Krehl, Arch. f. experim. Pathol. u. Pharmakol. 1895, S. 222; Patbologische Physiologie. Vogel, 1918. S. 80. - Kühne, Zeitschr. f. Biol. 1493. S. 221. -- Lindig. Münch. med. Wochenschr. 1919, Nr. 38 u. to. -... Mathes. Zentralbl. f, inn. Med. 1895. Nr. 16; Dtsch. Archiv f. klin. Med. 1845, S. 39. - Hayer. Zeitschr. f. experim. Pathol. u. Ther. 19. 1917; Zeitsehr. f. Juberkulose 1921, s. 6.21 (Elster). Moro. Münch. med. Wochenschr. 1920, Nr. 44. - Naegeli. Kraus Brugsch, Spez. Pathol. u. Ther. Urban und Schwarzenberg 8, \$. 64. 1920. -. Neufelt, Zeitschr. f. Tuberkulose 19 1, S. 606 (Elsteri. … v. Pirquet. Münch. med. Wochenschr. 1906; Nr. 30. - Ruppel, Münch. med. Wochenschr. 1910. Nr. 4ti; Zeitschr. f. physikal. (hemie $26 ; \ldots-$ und Josef, Zeitschr. f. Immunitätstorsch. u. exp. Therap. 1914. R. Schmidt, Med. Klin. 1919, Nr. 21. - E. Schulz, Beitr. z. Klin. d. Tuberkul. 21, 1911. - Selter, Zeitschr. f. Immunitätsforsch. u. exp. Therap. 1921. S. 32!; Dtsch. med. Wochenschr. 1921, Nr. 11. u. 19; Zeitschr. f. Tuberkulose 1921, S. 626 (Elster) - Sons und 2 . Mikulicz-Radechi. Dtsch. med. Wochensehr. 1921, Nr. 26. - v. Wassermann, Zeitschr. f. Tuberkulose 1921, S. 596 (Elster); - and Bruck, Dtsch. med. Wochenschr. 1906; Berl. klin. Wochenschr. 1907. - Weichardt, Zeitschr. f. Neurol. 22. H. 4 5. 1914; Münch. med. Wochenschr. 1915, Nr. 45; 1918, Nr. 29; 1919, Nr. 11; 19:0, Nr.4. IF eickel, Münch. med. Wochenschr. 1421. Nr. 30. - Ziegler, Verhandl des X. Kongr. f. inn. Med. 1491. - Zieler, Münch. med. Wochenschr. 1948. Nr. 32; 1)tsch. med. Wochenschr. 1911, Nr.45. - Zimmer, Therap. dl. Gegenw. 19.21), August. 